\title{
A Model System for Strong Interaction Between Internal Solitary Waves
}

\author{
Jerry L. Bona ${ }^{1,2}$, Gustavo Ponce ${ }^{1,5}$, Jean-Claude Saut ${ }^{3}$ \\ and Michael M. Tom ${ }^{1,4}$ \\ ${ }^{1}$ Department of Mathematics, The Pennsylvania State University, University Park, PA 16802, \\ USA \\ ${ }^{2}$ The Applied Research Laboratory, The Pennsylvania State University, University Park, \\ PA 16802, USA \\ ${ }^{3}$ Laboratoire d'Analyse Numérique, Université de Paris-Sud, Bâtiment 425, F-91405 Orsay \\ Cedex, France \\ ${ }^{4}$ Department of Mathematics, Louisiana State University, Baton Rouge, LA 70803, USA \\ 5 Department of Mathematics, University of California, Santa Barbara, CA 93106, USA
}

Received January 4, 1991; in revised form May 6, 1991

\begin{abstract}
A mathematical theory is mounted for a complex system of equations derived by Gear and Grimshaw that models the strong interaction of two-dimensional, long, internal gravity waves propagating on neighboring pycnoclines in a stratified fluid. For the model in question, the Cauchy problem is of interest, and is shown to be globally well-posed in suitably strong function spaces. Our results make use of Kato's theory for abstract evolution equations together with somewhat delicate estimates obtained using techniques from harmonic analysis. In weak function classes, a local existence theory is developed. The system is shown to be susceptible to the dispersive blow-up phenomenon investigated recently by Bona and Saut for Korteweg-de Vries-type equations.
\end{abstract}

\section{Introduction}

This paper is concerned with the initial-value problem

$$
\left\{\begin{array}{l}
u_{t}+u u_{x}+u_{x x x}+a_{3} v_{x x x}+a_{1} v v_{x}+a_{2}(u v)_{x}=0, \\
b_{1} v_{t}+r v_{x}+v v_{x}+v_{x x x}+b_{2} a_{3} u_{x x x}+b_{2} a_{2} u u_{x}+b_{2} a_{1}(u v)_{x}=0, \\
u(x, 0)=u_{0}(x), \\
v(x, 0)=v_{0}(x),
\end{array}\right.
$$

where $a_{1}, a_{2}, a_{3}, b_{1}, b_{2}$ and $r$ are real constants with $b_{1}, b_{2}$ positive, $u=u(x, t)$, $v=v(x, t)$ are real-valued functions of the two real variables $x$ and $t$, and subscripts 
adorning $u$ and $v$ connote partial differentiation. This somewhat complicated system has the structure of a pair of Korteweg-de Vries equations coupled through both dispersive and nonlinear effects. It was derived by Gear and Grimshaw (1984) as a model to describe the strong interaction of weakly nonlinear, long waves.

The model system (1.1) arises in the following general context. Consider a medium represented by $\mathbb{R} \times[-h, 0]$ in a standard Cartesian plane which can support two-dimensional wave motion in the horizontal direction. Frequently, the linearized theory for infinitesimal-amplitude wave motion in such a medium leads to a representation of a significant dependent variable $\eta$, say, in the form $\eta=A(x, t) \phi_{k}(y)$, where $x$ is the variable in the horizontal direction, $y$ the vertical variable and $\phi_{k}$ is an eigenfunction of some linear eigenvalue problem posed for $y \in[-h, 0]$, and with appropriate boundary conditions at $y=-h$ and $y=0$, $k=1,2, \ldots$. Different motions are associated to different modes $\phi_{k}$ which define the particular vertical structure of the wave, though often interest is focussed on one of the lowest modes. In this sort of representation, $A=A_{k}$ is a function of $x-c_{k} t$, where $c_{k}$ is the eigenvalue associated to the eigenfunction $\phi_{k}, k=1,2, \ldots$. When the theory is extended to allow for the weak effects of nonlinearity and dispersion, $\eta$ is represented in the form $\varepsilon A_{k} \phi_{k}$, where $\varepsilon$ is a small, amplitude parameter and $\phi_{k}$ is as before. Such a form is based on substantial assumptions about the spatial and temporal scales appropriate to the wave motion. Usually $A$ is a function of a long spatial variable $\varepsilon^{\alpha} x$ and a slow time variable perturbation $\varepsilon^{\beta} t$ of the basic speed $c_{k}$ associated to the mode $\phi_{k}$, where $\alpha$ and $\beta$ are positive constants that reflect the particular laws governing the motion. The function $A$ is then seen to satisfy a nonlinear partial differential equation and the combination $\varepsilon A \phi_{k}$ is taken to be a good approximation to the underlying wave motion on a longer time scale than provided by the corresponding solution of the linear equation.

An interesting possibility now presents itself, in which a motion may be initiated in the medium which corresponds to more than one of the vertical modes $\phi_{k}$. Consider the case wherein there are two different modes $\phi_{k}$ and $\phi_{m}$ and the motions associated with each are localized in space. If the fundamental phase speeds $c_{k}$ and $c_{m}$ associated with these modes differ sufficiently, then basically the motions associated with each will pull apart rapidly enough that, to leading order in the parameter $\varepsilon, A_{k}$ and $A_{m}$ are determined independently of one another. However, if $c_{k}$ and $c_{m}$ differ by order $\varepsilon^{\beta}$, there is the prospect that the motions associated with $\phi_{k}$ and $\phi_{m}$ may remain in the spatial vicinity of each other long enough that the effect of interaction between them can accumulate to make a leading-order difference to each amplitude function $A_{k}$ and $A_{m}$. In this case, $A_{k}$ and $A_{m}$ will satisfy a coupled system of partial differential equations. It is to this latter, interesting situation in the nonlinear regime that the present work is devoted.

Consider now the concrete situation of wave motion in a density-stratified fluid of constant total depth $h$ which is far from any lateral boundary. Assuming the motion to be uniform in one of the unbounded directions, and neglecting dissipative effects, the two-dimensional Euler equations are taken to be the full equations of motion. If the undisturbed density variation is a function $\rho_{0}=\rho_{0}(y)$ of the vertical coordinate alone, then we find that the generalities outlined above apply. In 
particular, the linear eigenvalue problem for the vertical modes is

$$
\begin{cases}c_{k}^{2} \frac{\partial}{\partial y}\left(\rho_{0} \frac{\partial \phi_{k}}{\partial y}\right)+\rho_{0} N^{2} \phi_{k}=0 & \text { for } \quad 0>y>-h \\ \phi_{k}=0 & \text { on } y=-h, \\ \phi_{k}=\gamma c_{k}^{2} \frac{\partial \phi_{k}}{\partial y} & \text { on } y=0,\end{cases}
$$

where $N^{2}(y)$ is the Brunt-Väisälä frequency (and so proportional to $\rho_{0 y}$ ) and $\gamma$ is either zero in case the upper boundary is fixed or is the Boussinesq parameter (a non-dimensional measure of $g^{-1}$, where $g$ is the gravity constant) if the upper surface is free. Erkart (1961) examined the linearized problem in the case where the undisturbed density variation $\rho_{0}$ consists of two, well separated pycnoclines, and determined that resonant transfer of energy between waves propagating on each of the two pycnoclines is possible when the waves in question have nearly identical phase speeds. In the same configuration, Liu, Kubota and Ko (1980) and Liu, Pereira and Ko (1982) showed that such energy transfer was possible between nonlinear waves propagating on widely separated, neighboring pycnoclines. In particular, they found that solitary waves propagating on neighboring pycnoclines could interact strongly. The model with which they drew these conclusions consists of a pair of intermediate depth equation (cf. Kubota, Ko and Dobbs 1980) coupled through a purely dispersive term. This system has recently been analyzed by Bona and Saut (1991b). In contrast, when the overall depth $h$ of the fluid is shallow with regard to a typical wavelength $L$, so that, in particular, neighboring pycnoclines are not widely separated, Gear and Grimshaw (1984) have shown that the strong or resonant interaction between waves on neighboring pycnoclines is approximately governed by the pair of Korteweg-de Vries (K-dV) equations in (1.1) which are coupled through both nonlinear and dispersive effects. Indeed, the assumptions leading to (1.1) include that $h^{2} / L^{2}$ is of the same order as the amplitude $\varepsilon$, and this plus the presumption of one-way propagation leads inevitably to a $\mathrm{K}$-dV-type model.

If $\phi_{n}$ and $\phi_{m}$ are two distinct solutions of the eigenvalue problem (1.2) with phase speeds $c_{n}$ and $c_{m}$ differing by a quantity proportional to $\varepsilon$, i.e. $c_{m}=c_{n}-\varepsilon \chi$, then Gear and Grimshaw found that the vertical displacement $\eta=\eta(\theta, y, \tau)$ of the fluid is given by

$$
\eta=\varepsilon\left\{A_{n}(\tau, \theta) \phi_{n}(y)+A_{m}(\tau, \theta) \phi_{m}(y)\right\}+O\left(\varepsilon^{2}\right),
$$

where the wave amplitudes $A_{n}$ and $A_{m}$ satisfy the evolution equations

$$
\begin{aligned}
0= & 2 \frac{I_{n}}{c_{n}^{2}}\left(\frac{1}{c_{n}} \frac{\partial A_{n}}{\partial \tau}+\mu_{n} A_{n} \frac{\partial A_{n}}{\partial \theta}+\lambda_{n} \frac{\partial^{3} A_{n}}{\partial \theta^{3}}\right) \\
& +3 v_{n m m} A_{m} \frac{\partial A_{m}}{\partial \theta}+3 v_{n n m} \frac{\partial}{\partial \theta}\left(A_{n} A_{m}\right)+\lambda_{n m} \frac{\partial^{3} A_{m}}{\partial \theta^{3}}
\end{aligned}
$$


and

$$
\begin{aligned}
0= & 2 \frac{I_{m}}{c_{n}^{2}}\left(\frac{1}{c_{n}} \frac{\partial A_{m}}{\partial \tau}-\frac{\chi}{c_{n}} \frac{\partial A_{m}}{\partial \theta}+\mu_{m} A_{m} \frac{\partial A_{m}}{\partial \theta}+\lambda_{m} \frac{\partial^{3} A_{m}}{\partial \theta^{3}}\right) \\
& +3 v_{n m m} A_{n} \frac{\partial A_{n}}{\partial \theta}+3 v_{n m m} \frac{\partial}{\partial \theta}\left(A_{n} A_{m}\right)+\lambda_{n m} \frac{\partial^{3} A_{n}}{\partial \theta^{3}}
\end{aligned}
$$

with

$$
\begin{aligned}
v_{s k l} & =\int_{-h}^{0} \rho_{0} \frac{\partial \phi_{s}}{\partial y} \frac{\partial \phi_{k}}{\partial y} \frac{\partial \phi_{l}}{\partial y} d y \\
\lambda_{k l} & =\int_{-h}^{0} \rho_{0} \phi_{k} \phi_{l} d y \\
I_{k} \mu_{k} & =\frac{3}{2} c_{k}^{2} \int_{-h}^{0} \rho_{0}\left(\frac{\partial \phi_{k}}{\partial y}\right)^{3} d y \\
I_{k} \lambda_{k} & =\frac{1}{2} c_{k}^{2} \int_{-h}^{0} \rho_{0} \phi_{k}^{2} d y
\end{aligned}
$$

and the orthogonality condition

$$
\delta_{k l} I_{k}=c_{k}^{2} \int_{-h}^{0} \rho_{0} \frac{\partial \phi_{k}}{\partial y} \frac{\partial \phi_{l}}{\partial y} d y,
$$

where $\delta_{k l}$ is the Kronecker delta. Note that $2 I_{k} \mu_{k}=3 c_{k}^{2} v_{k k k}$ and $2 I_{k} \lambda_{k}=c_{k}^{2} \lambda_{k k}$. Now by letting

$$
\begin{aligned}
A_{n} & =\frac{\chi \lambda_{n}}{r \lambda_{m} \mu_{n}} u, \quad A_{m}=\frac{\chi}{r \mu_{m}} v, \\
\theta & =\left(\frac{r \lambda_{m}}{\chi}\right)^{1 / 2} x, \quad \tau=\left(\frac{r \lambda_{m}}{\chi}\right)^{3 / 2} \frac{t}{c_{n} \lambda_{n}},
\end{aligned}
$$

the system (1.1) is recovered with the constants $a_{1}, a_{2}, a_{3}, b_{1}, b_{2}$ given by

$$
\begin{aligned}
& a_{1}=\frac{3 c_{n}^{2} v_{n m m} \mu_{n} \lambda_{m}^{2}}{2 I_{n} \mu_{m}^{2} \lambda_{n}^{2}}, \quad a_{2}=\frac{3 c_{n}^{2} v_{n n m} \lambda_{m}}{2 I_{n} \mu_{m} \lambda_{n}}, \quad a_{3}=\frac{c_{n}^{2} \mu_{n} \lambda_{n m} \lambda_{m}}{2 I_{n} \mu_{m} \lambda_{n}^{2}}, \\
& b_{1}=\frac{\lambda_{n}}{\lambda_{m}}, \quad b_{2}=\frac{I_{n} \lambda_{n}^{3} \mu_{m}^{2}}{I_{m} \lambda_{m}^{3} \mu_{n}^{2}}
\end{aligned}
$$

The constant $r$ is a non-dimensional, disposable parameter which does not affect our analysis and which will henceforth be neglected. Notice that the parameters $b_{1}$ and $b_{2}$ are automatically positive since $I_{n}$ and $\lambda_{n}$ are strictly positive because the density is strictly positive and the $\phi_{k}$ are not constant in the presence of true stratification $\left(\rho_{0 y} \neq \equiv\right)$.

Gear (1985) showed that although these coupled equations possess an exact, traveling-wave solution involving the characteristic $\operatorname{sech}^{2}$-profile of the $\mathrm{K}-\mathrm{dV}$ equation, they are in general not solvable by the inverse-scattering transform technique. 
Our aim in this article is to study local and global well-posedness of the initial-value problem (1.1) in the classical Sobolev spaces $H^{s}(\mathbb{R}) \times H^{s}(\mathbb{R})$. The problem (1.1) is said to be locally well-posed in $H^{s}(\mathbb{R}) \times H^{s}(\mathbb{R})$ if it generates a continuous local flow in $H^{s}(\mathbb{R}) \times H^{s}(\mathbb{R})$ (i.e. if existence, uniqueness, persistence, and continuous dependence on the initial data hold). The problem is globally well-posed if the local flow can be continued indefinitely in the temporal variable, so defining a solution of (1.1) valid for all $t \geqq 0$. Such a theory is basic to the analytical or numerical study of the system.

It will be demonstrated that (1.1) is locally well-posed in $H^{s}(\mathbb{R}) \times H^{s}(\mathbb{R})$ for any $s \geqq 2$. As with other, dispersive wave equations, global well-posedness in any particular Sobolev space seems to depend on the available local theory and on the conservation laws or energy-type inequalities satisfied by the solutions. In general, solutions of (1.1) satisfy the following conservation laws:

$$
\Phi_{1}(u)=\int_{-\infty}^{\infty} u d x, \quad \Phi_{2}(v)=\int_{-\infty}^{\infty} v d x, \quad \Phi_{3}(u, v)=\int_{-\infty}^{\infty}\left(b_{2} u^{2}+b_{1} v^{2}\right) d x
$$

The time-invariance of the functionals $\Phi_{1}$ and $\Phi_{2}$ expresses the property that the mass of each mode separately is conserved during iteraction, while that of $\Phi_{3}$ is an expression of the conservation of energy for the system of two modes taken as a whole. In general, while total energy is conserved, it may nevertheless be passed between the two modes. As remarked by Liu, Kubota and Ko (1980) in a related problem, the functional $\Phi_{3}$ supports the tentative conclusion that a mode can increase its energy only by increasing its amplitude and decreasing its width, while the other mode must correspondingly decrease its energy by decreasing its amplitude and increasing its width. Solutions of (1.1) satisfy an additional conservation law which is revealed by the time-invariance of the functional

$$
\Phi_{4}(u, v)=\int_{-\infty}^{\infty}\left(b_{2} u_{x}^{2}+v_{x}^{2}+2 b_{2} a_{3} u_{x} v_{x}-b_{2} \frac{u^{3}}{3}-b_{2} a_{2} u^{2} v-b_{2} a_{1} u v^{2}-\frac{v^{3}}{3}-r v^{2}\right) d x
$$

The functional $\Phi_{4}$ is a Hamiltonian for the system (1.1), and if $b_{2} a_{3}^{2}<1, \Phi_{4}$ will be seen to provide an a priori estimate for the solution pair $(u, v)$ in the space $H^{1}(\mathbb{R}) \times H^{1}(\mathbb{R})$. Furthermore, the linearization of $(1.1)$ about the rest state can be reduced to two, linear $\mathrm{K}-\mathrm{dV}$ equations by a process of diagonalization. Using this remark and the smoothing properties (in both the temporal and spatial variables) for the linear K-dV derived by Kato $(1975,1979,1983)$ Kenig, Ponce and Vega $(1989,1991 \mathrm{a}, \mathrm{b})$, it will be shown that (1.1) is locally well-posed in $H^{s}(\mathbb{R}) \times H^{s}(\mathbb{R})$ for any $s \geqq 1$ if $\sqrt{b_{2}} a_{3} \neq \pm 1$. It will therefore follow from the a priori estimates provided by $\Phi_{3}$ and $\Phi_{4}$ that the system (1.1) is globally well-posed in $H^{s}(\mathbb{R}) \times H^{s}(\mathbb{R})$ for any $s \geqq 1$ whenever $\left|a_{3}\right|<1 / \sqrt{b_{2}}$.

It is worth contrasting the theory developed here for (1.1) with the early methods used to prove global well-posedness for the K-dV equation in the spaces $H^{k}(\mathbb{R})$ for $k \geqq 2$ (see Bona and Smith 1975; Kato 1975; Saut and Temam 1976). These theories relied on an a priori bound in $H^{2}(\mathbb{R})$ provided by one of the infinite string of conservation laws with polynomial densities. For the system (1.1), we have only found the four conservation laws listed above, and these only provide a priori information in $H^{1}(\mathbb{R}) \times H^{1}(\mathbb{R})$. Hence, to establish global well-posedness, it is 
central to our argument that (1.1) is locally well-posed in the relatively weak space $H^{1}(\mathbb{R}) \times H^{1}(\mathbb{R})$. In consequence of this requirement, we must call on the sort of theory developed recently in Kenig, Ponce and Vega (1991b) for the initial-value problem for the K-dV equation posed in the space $H^{s}(\mathbb{R})$ for smallish values of $s$.

This paper is organized as follows. The general, local well-posedness result together with a sufficient condition for local solutions to be globally continuable are presented in Sect. 2, while a priori estimates in $H^{1}(\mathbb{R}) \times H^{1}(\mathbb{R})$ are derived in Sect. 3. Estimates concerning the linear propagator are contained in Sect. 4, and our main result for smooth solution is established in Sect. 5. In addition to the theory of strong solutions, we are also able to develop existence results in case the initial data lies only in $L^{2}(\mathbb{R}) \times L^{2}(\mathbb{R})$. This theory of weak solutions is coupled with a theory of existence in weighted Sobolev spaces to demonstrate a type of singularity formation termed dispersive blow up in Bona and Saut (1991a). These results, which appear in Sect. 6, are a result of the way dispersion appears in the model, with a negatively unbounded group and phase velocity.

Notation. The norm in $L^{p}(\mathbb{R}), 1 \leqq p \leqq \infty$ will be denoted by $\|\cdot\|_{p}$. We shall use the abbreviations $J^{s}=\left(1-\partial_{x}^{2}\right)^{s / 2}$ and $D^{s}=\left(-\partial_{x}^{2}\right)^{s / 2}$ to denote the Bessel and Riesz potentials of order $-s$, respectively. Define $L_{s}^{p}=J^{-s} L^{p}$, a Banach space whose norm will be denoted by $\|\cdot\|_{s, p}=\left\|J^{s} \cdot\right\|_{p}$. When $p=2, L_{s}^{2}$ is the classical Sobolev space $H^{s}(\mathbb{R})$, and $H^{\infty}(\mathbb{R})=\bigcap_{s>0} H^{s}(\mathbb{R})$. Also define the commutator between two operators $A$ and $B$ by $[A, B]=A B-B A$. Thus, $\left[J^{s}, f\right] g=J^{s}(f g)-f J^{s} g$ in which $f$ is regarded as a multiplication operator. The space $H_{\text {loc }}^{s}(\Omega)$, where $\Omega$ is an open set in $\mathbb{R}$ and $s \geqq 0$ connotes the class of measurable functions $f$ defined on $\Omega$ such that for every $\phi \in C_{0}^{\infty}(\Omega), \phi f \in H^{s}(\mathbb{R})$. If $[0, T]$ is an interval and $X$ is a Banach space with norm $\|\cdot\|_{X}$, then

$$
L^{p}(0, T ; X)=\left\{u:[0, T] \rightarrow X \text { such that } \int_{0}^{T}\|u\|_{X}^{p}<\infty\right\} .
$$

The space $C(0, T ; X)$ comprises the class of all continuous functions mapping $[0, T]$ into $X$. If $[0, T]$ is compact, $C(0, T ; X)$ is a Banach space when equipped with the norm $\|\cdot\|_{L^{\infty}(0, T ; X)}$.

\section{General Local Theory}

In this section, we shall present a theorem about the local well-posedness of the initial-value problem (1.1) and a continuation principle that ensures local solutions to be extendable to smooth solutions defined globally in time. The well-posedness theorem is obtained by a straightforward application of the abstract techniques of Kato $(1975,1983)$ for quasi-linear evolution equations and hence the proof is abbreviated. The continuation principle uses the local theory and energy-type estimates.

For simplicity of exposition, we shall restrict ourselves to integer-order Sobolev spaces. This is a restriction of convenience only. For any $T>0$ a finite number and $s$ an arbitrary integer Sobolev index, let

$$
X_{s}(T)=C\left(0, T ; H^{s}(\mathbb{R})\right) \cap C^{1}\left(0, T ; H^{s-3}(\mathbb{R})\right) .
$$


Theorem 2.1. Let $s \geqq 2$ be an integer, and $\left(u_{0}, v_{0}\right) \in H^{s}(\mathbb{R}) \times H^{s}(\mathbb{R})$. Then there exists a $T=T\left(\left\|\left(u_{0}, v_{0}\right)\right\|_{s, 2}\right)>0$ and a unique solution $(u, v) \in X_{s}(T) \times X_{s}(T)$ of the system (1.1) corresponding to the initial data $\left(u_{0}, v_{0}\right)$. Moreover, the pair $(u, v)$ depends continuously on $\left(u_{0}, v_{0}\right)$ in the sense that the mapping $\left(u_{0}, v_{0}\right) \mapsto(u, v)$ is continuous from $H^{s}(\mathbb{R}) \times H^{s}(\mathbb{R})$ into the space $X_{s}(T) \times X_{s}(T)$.

As mentioned above, Theorem 2.1 is an easy consequence of the general results of Kato. The functional-analytic setting for Kato's theory consists of a pair of reflexive Banach spaces $X$ and $Y$, where $Y \subset X$ with the injection continuous and dense. A central role in the theory is played by a Banach-space isomorphism $S$ of $Y$ onto $X$, and the norms on these two spaces are chosen in such a way that $S$ is an isometry. The theory applies to the abstract, quasi-linear evolution equation

$$
U_{t}+A(t, U) U=F(t, U)
$$

for $0<t$, with

$$
U(0)=U_{0},
$$

where $U_{0} \in Y$ is a given initial value. Kato's theory asserts that there exists a positive time $T=T\left(\left\|U_{0}\right\|_{Y}\right)$ such that (2.1) possesses a unique solution in $C(0, T ; Y) \cap C^{1}(0, T ; X)$ provided that certain conditions are satisfied. Moreover, the mapping that associates to $U_{0}$ the solution $U$ is continuous from $Y$ into $C(0, T ; Y) \cap C^{1}(0, T ; X)$.

To apply this theory to the situation of interest here in case $s \geqq 3$, take $X=H^{s-3}(\mathbb{R}) \times H^{s-3}(\mathbb{R})$ and $Y=H^{s}(\mathbb{R}) \times H^{s}(\mathbb{R})$, let $S=\left(J^{3}, J^{3}\right)$, let $A$ be the matrix operator

$$
A=A(W)=\left(\begin{array}{cc}
\partial_{x}^{3}+y \partial_{x}+a_{2} z \partial_{x} & a_{3} \partial_{x}^{3}+a_{1} z \partial_{x}+a_{2} y \partial_{x} \\
\frac{b_{2} a_{3}}{b_{1}} \partial_{x}^{3}+\frac{b_{2} a_{2}}{b_{1}} y \partial_{x}+\frac{b_{2} a_{1}}{b_{1}} z \partial_{x} & \frac{1}{b_{1}} \partial_{x}^{3}+\frac{1}{b_{1}} z \partial_{x}+\frac{b_{2} a_{1}}{b_{1}} y \partial_{x}
\end{array}\right),
$$

where

$$
W=\left(\begin{array}{l}
y \\
z
\end{array}\right),
$$

and let the operator $F$ be zero. With this choice of $A$ and $F$, and writing

$$
U=\left(\begin{array}{l}
u \\
v
\end{array}\right),
$$

(1.1) reduces to (2.1), if $U_{0}=\left(u_{0}, v_{0}\right)$. To verify the hypotheses of Kato's theory, it is convenient to take the inner product of two elements $(f, g)$ and $(\eta, h)$ in $L^{2}(\mathbb{R}) \times L^{2}(\mathbb{R})$ to be

$$
\langle(f, g),(\eta, h)\rangle=b_{2} \int_{-\infty}^{\infty} f \eta+b_{1} \int_{-\infty}^{\infty} g h
$$

Hence the norm of an element $(f, g) \in H^{r}(\mathbb{R}) \times H^{r}(\mathbb{R})$, where $r \geqq 0$ will be given by

$$
\|(f, g)\|_{r, 2}=\left(b_{2}\|f\|_{r, 2}^{2}+b_{1}\|g\|_{r, 2}^{2}\right)^{1 / 2},
$$


where as mentioned above, $b_{1}$ and $b_{2}$ are known to be positive. It is then straightforward to verify that the hypotheses of Kato's theory are satisfied.

In case $s<3$, one takes $X=L^{2}(\mathbb{R}) \times L^{2}(\mathbb{R}), Y=H^{s}(\mathbb{R}) \times H^{s}(\mathbb{R})$, and $S=\left(J^{s}, J^{s}\right)$. To apply the theory, one needs to use the trick observed by Kato (1979) in which new variables $\tilde{u}$ and $\tilde{v}$ are defined by conjugating $u, v$ with $e^{t \partial_{x}^{3}}$, viz

$$
\tilde{u}=e^{-t \partial_{x}^{3}} u e^{t \partial_{x}^{3}}
$$

The details follow exactly the lines laid down by Kato, and so they are passed over here.

Lemma 2.2. If $(u, v) \in X_{s}(T) \times X_{s}(T)$ is a local solution of the initial value problem (1.1) corresponding to the initial data $\left(u_{0}, v_{0}\right)$ as specified by Theorem 2.1, then for any $k$ with $1 \leqq k \leqq s$,

$$
\sup _{[0, T]}\|(u(\cdot, t), v(\cdot, t))\|_{k, 2} \leqq\left\|\left(u_{0}, v_{0}\right)\right\|_{k, 2} \exp \left(C \int_{0}^{T}\left(\left\|u_{x}(\cdot, \tau)\right\|_{\infty}+\left\|v_{x}(\cdot, \tau)\right\|_{\infty}\right) d \tau\right) .
$$

Proof. In light of the local well-posedness theory sketched above, the following formal calculations can be easily justified provided $k \leqq s$ by simply regularizing the initial data, making the calculations for the associated smooth solutions, and then passing to the limit in which the regularization is allowed to degenerate to the identity operator. Consider the case $k=1$. Differentiate the first equation in (1.1) with respect to $x$, multiply by $u_{x}$ and integrate the resulting expression over $\mathbb{R}$, thereby obtaining the equation

$$
\frac{1}{2} \frac{d}{d t} \int_{-\infty}^{\infty} u_{x}^{2}+a_{3} \int_{-\infty}^{\infty} u_{x} v_{x x x x}=-\frac{1}{2} \int_{-\infty}^{\infty} u^{2} u_{x x x}-\frac{a_{1}}{2} \int_{-\infty}^{\infty} v^{2} u_{x x x}-a_{2} \int_{-\infty}^{\infty} u v u_{x x x} .
$$

Now, divide the second equation in (1.1) by $b_{2}$ and perform calculations similar to those leading to (2.3) to arrive at the relation

$$
\begin{aligned}
\frac{b_{1}}{2 b_{2}} \frac{d}{d t} \int_{-\infty}^{\infty} v_{x}^{2}+a_{3} \int_{-\infty}^{\infty} v_{x} u_{x x x x}= & -\frac{1}{2 b_{2}} \int_{-\infty}^{\infty} v^{2} v_{x x x} \\
& -\frac{a_{2}}{2} \int_{-\infty}^{\infty} u^{2} v_{x x x}-a_{1} \int_{-\infty}^{\infty} u v v_{x x x} .
\end{aligned}
$$

Adding (2.3) and (2.4) leads to the equation

$$
\begin{aligned}
\frac{1}{2} \frac{d}{d t} \int_{-\infty}^{\infty}\left(b_{2} u_{x}^{2}+b_{1} v_{x}^{2}\right)= & -\frac{b_{2}}{2} \int_{-\infty}^{\infty} u^{2} u_{x x x}-\frac{a_{1} b_{2}}{2} \int_{-\infty}^{\infty} v^{2} u_{x x x} \\
& -a_{2} b_{2} \int_{-\infty}^{\infty} u v u_{x x x}-\frac{1}{2} \int_{-\infty}^{\infty} v^{2} v_{x x x} \\
& -\frac{a_{2} b_{2}}{2} \int_{-\infty}^{\infty} u^{2} v_{x x x}-a_{1} b_{2} \int_{-\infty}^{\infty} u v v_{x x x}
\end{aligned}
$$

from which one obtains the inequalities

$$
\frac{1}{2} \frac{d}{d t} \int_{-\infty}^{\infty}\left(b_{2} u_{x}^{2}+b_{1} v_{x}^{2}\right)=-\frac{b_{2}}{2} \int_{-\infty}^{\infty} u_{x}^{3}-\frac{1}{2} \int_{-\infty}^{\infty} v_{x}^{3}
$$




$$
\begin{aligned}
& -\frac{3 a_{2} b_{2}}{2} \int_{-\infty}^{\infty} u_{x}^{2} v_{x}-\frac{3 a_{1} b_{2}}{2} \int_{-\infty}^{\infty} v_{x}^{2} u_{x} \\
\leqq & C\left(\left\|u_{x}\right\|_{\infty}+\left\|v_{x}\right\|_{\infty}\right)\left(\left\|u_{x}\right\|_{2}^{2}+\left\|v_{x}\right\|_{2}^{2}\right) \\
\leqq & C\left(\left\|u_{x}\right\|_{\infty}+\left\|v_{x}\right\|_{\infty}\right)\left(b_{2}\left\|u_{x}\right\|_{2}^{2}+b_{1}\left\|v_{x}\right\|_{2}^{2}\right) .
\end{aligned}
$$

Now consider the case $k \geqq 2$. Apply $\partial_{x}^{k}$ to both sides of the first equation in (1.1), multiply this expression by $\partial_{x}^{k} u$ and integrate the resulting expression over $\mathbb{R}$. One finds that

$$
\begin{aligned}
\frac{1}{2} \frac{d}{d t} \int_{-\infty}^{\infty}\left(\partial_{x}^{k} u\right)^{2}+a_{3} \int_{-\infty}^{\infty} \partial_{x}^{k} u \partial_{x}^{k} v_{x x x}= & -\int_{-\infty}^{\infty} \partial_{x}^{k} u \partial_{x}^{k}\left(u u_{x}\right) \\
& -a_{1} \int_{-\infty}^{\infty} \partial_{x}^{k} u \partial_{x}^{k}\left(v v_{x}\right)-a_{2} \int_{-\infty}^{\infty} \partial_{x}^{k} u \partial_{x}^{k}(u v)_{x}
\end{aligned}
$$

Next, divide the second equation in (1.1) by $b_{2}$ and perform a similar set of operations to obtain

$$
\begin{aligned}
& \frac{b_{1}}{2 b_{2}} \frac{d}{d t} \int_{-\infty}^{\infty}\left(\partial_{x}^{k} v\right)^{2}+a_{3} \int_{-\infty}^{\infty} \partial_{x}^{k} v \partial_{x}^{k} u_{x x x} \\
& \quad=-\frac{1}{b_{2}} \int_{-\infty}^{\infty} \partial_{x}^{k} v \partial_{x}^{k}\left(v v_{x}\right)-a_{1} \int_{-\infty}^{\infty} \partial_{x}^{k} v \partial_{x}^{k}(u v)_{x}-a_{2} \int_{-\infty}^{\infty} \partial_{x}^{k} v \partial_{x}^{k}\left(u u_{x}\right) .
\end{aligned}
$$

Adding (2.7) and (2.8), using Leibniz's rule and several integrations by parts together with Hölder's inequality, one obtains the estimates

$$
\begin{aligned}
\frac{d}{d t}\left(b_{2}\left\|\partial_{x}^{k} u\right\|_{2}^{2}+b_{1}\left\|\partial_{x}^{k} v\right\|_{2}^{2}\right) & \leqq C\left(\left\|u_{x}\right\|_{\infty}+\left\|v_{x}\right\|_{\infty}\right)\left(\left\|\partial_{x}^{k} u\right\|_{2}^{2}+\left\|\partial_{x}^{k} v\right\|_{2}^{2}\right) \\
& \leqq C\left(\left\|u_{x}\right\|_{\infty}+\left\|v_{x}\right\|_{\infty}\right)\left(b_{2}\left\|\partial_{x}^{k} u\right\|_{2}^{2}+b_{1}\left\|\partial_{x}^{k} v\right\|_{2}^{2}\right)
\end{aligned}
$$

Gronwall's inequality applied to (2.6) and (2.9) yields the desired results.

Remark 2.1. Actually Theorem 2.1 is valid for fractional exponents $s>3 / 2$, while Lemma 2.2 is valid for fractional indices $s \geqq 0$. However, to prove the latter assertion, we seem to need the commutator-estimates of Kato and Ponce (1988) and so this level of generality has been eschewed in favor of the simpler proof presented above.

Lemma 2.3. Let $\left(u_{0}, v_{0}\right) \in H^{s}(\mathbb{R}) \times H^{s}(\mathbb{R})$, where $s \geqq 2$ and let $(u, v)$ be the local solution as in Theorem 2.1 emanating therefrom. Suppose there are finite constants $K$ and $T_{1}$ such that for any $t \leqq T_{1}$ for which the solution exists on the interval $[0, t]$, we have

$$
\int_{0}^{t}\left(\left\|u_{x}(\cdot, r)\right\|_{\infty}+\left\|v_{x}(\cdot, r)\right\|_{\infty}\right) d r \leqq K .
$$

Then the local solution can be extended at least over the time interval $\left[0, T_{1}\right]$.

Proof. Let $T_{0}$ be the maximum temporal existence interval for the solution $(u, v)$ and suppose $T_{0} \leqq T_{1}$. Thus for any $T<T_{0},(u, v) \in X_{s}(T) \times X_{s}(T)$, and so from (2.2) 
and (2.10) it follows that for any such $T$, we have

$$
\sup _{[0, T]}\|(u(t), v(t))\|_{s, 2} \leqq\left\|\left(u_{0}, v_{0}\right)\right\|_{s, 2} \exp (C K),
$$

for any $s \geqq 1$. Hence there is a uniform bound on $(u, v)$ in $H^{s}(\mathbb{R}) \times H^{s}(\mathbb{R})$ on the time interval $[0, T)$. This fact and the local existence theory contradict the definition of $T_{0}$ unless $T_{0}>T_{1}$.

\section{A Priori Estimates in $H^{1}(\mathbb{R}) \times H^{1}(\mathbb{R})$}

The conservation laws $\Phi_{1}, \Phi_{2}$ and $\Phi_{3}$ were derived by Gear and Grimshaw (1984). The Hamiltonian functional $\Phi_{4}$ in (1.5) appears to be new and hence its derivation is offered now.

Multiply Eq. (2.3) by $b_{1} b_{2}$ to obtain

$$
\begin{aligned}
& \frac{b_{1} b_{2}}{2} \frac{d}{d t} \int_{-\infty}^{\infty} u_{x}^{2}+b_{1} b_{2} a_{3} \int_{-\infty}^{\infty} u_{x} v_{x x x x} \\
& \quad=-\frac{b_{1} b_{2}}{2} \int_{-\infty}^{\infty} u^{2} u_{x x x}-\frac{b_{1} b_{2} a_{1}}{2} \int_{-\infty}^{\infty} v^{2} u_{x x x}-b_{1} b_{2} a_{2} \int_{-\infty}^{\infty} u v u_{x x x} .
\end{aligned}
$$

Differentiating the first equation in (1.1) with respect to $x$, but this time multiplying by $b_{1} b_{2} a_{3} v_{x}$ and integrating over $\mathbb{R}$, we find that

$$
\begin{aligned}
& a_{3} b_{1} b_{2} \int_{-\infty}^{\infty} v_{x} u_{x t}+a_{3} b_{1} b_{2} \int_{-\infty}^{\infty} v_{x} u_{x x x x} \\
& =\frac{a_{3} b_{1} b_{2}}{2} \int_{-\infty}^{\infty} u^{2} v_{x x x}-\frac{a_{1} a_{3} b_{1} b_{2}}{2} \int_{-\infty}^{\infty} v^{2} v_{x x x}-a_{2} a_{3} b_{1} b_{2} \int_{-8}^{\infty} u v v_{x x x} .
\end{aligned}
$$

A similar operation applied to the second equation in (1.1) gives

$$
\begin{aligned}
& a_{3} b_{1} b_{2} \int_{-\infty}^{\infty} u_{x} v_{x t}+a_{3} r b_{2} \int_{-\infty}^{\infty} v_{x x} u_{x}+a_{3} b_{2} \int_{-\infty}^{\infty} u_{x} v_{x x x x} \\
& =-\frac{a_{3} b_{2}}{2} \int_{-\infty}^{\infty} v^{2} u_{x x x}-\frac{a_{2} a_{3} b_{2}^{2}}{2} \int_{-\infty}^{\infty} u^{2} u_{x x x}-a_{1} a_{3} b_{2}^{2} \int_{-\infty}^{\infty} u v u_{x x x} .
\end{aligned}
$$

Adding (2.4), (3.1), (3.2) and (3.3), we find that

$$
\begin{aligned}
& \frac{b_{1}}{2} \frac{d}{d t} \int_{-\infty}^{\infty}\left(b_{2} u_{x}^{2}+v_{x}^{2}+2 a_{3} b_{2} u_{x} v_{x}\right) \\
& =-\frac{b_{1} b_{2}}{2} \int_{-\infty}^{\infty} u^{2}\left(u_{x x x}+a_{3} v_{x x x}\right)-\frac{a_{1} b_{1} b_{2}}{2} \int_{-\infty}^{\infty} v^{2}\left(u_{x x x}+a_{3} v_{x x x}\right) \\
& \quad-a_{2} b_{1} b_{2} \int_{-\infty}^{\infty} u v\left(u_{x x x}+a_{3} v_{x x x}\right)-\frac{1}{2} \int_{-\infty}^{\infty} v^{2}\left(v_{x x x}+a_{3} b_{2} u_{x x x}\right) \\
& \quad-\frac{a_{2} b_{2}}{2} \int_{-\infty}^{\infty} u^{2}\left(v_{x x x}+a_{3} b_{2} u_{x x x}\right)-a_{1} b_{2} \int_{-\infty}^{\infty} u v\left(v_{x x x}+a_{3} b_{2} u_{x x x}\right) \\
& \quad-r a_{3} b_{2} \int_{-\infty}^{\infty} v u_{x x x} .
\end{aligned}
$$


Finally, using the original Eqs. (1.1) in (3.4) gives

$$
\frac{b_{1}}{2} \frac{d}{d t} \int_{-\infty}^{\infty}\left(b_{2} u_{x}^{2}+v_{x}^{2}+2 a_{3} b_{2} u_{x} v_{x}-b_{2} \frac{u^{3}}{3}-b_{2} a_{2} u^{2} v-b_{2} a_{1} u v^{2}-\frac{v^{3}}{3}-r v^{2}\right) d x=0
$$

which says that $\Phi_{4}$ is time-invariant when evaluated on a solution pair $(u, v)$ of $(1.1)$.

Lemma 3.1. Let $(u, v) \in X_{s}(T) \times X_{s}(T)$ be the solution to (1.1) corresponding to the initial data $\left(u_{0}, v_{0}\right)$ in $H^{s}(\mathbb{R}) \times H^{s}(\mathbb{R})$, where $s \geqq 2$.

(1) Then $\|(u(t), v(t))\|_{2}$ is bounded independently of $t$, with a bound depending only on $\left\|\left(u_{0}, v_{0}\right)\right\|_{2}$.

(2) If $\left|a_{3}\right|<1 / \sqrt{b_{2}}$, then $\|(u(t), v(t))\|_{1,2}$ is bounded independently of $t$, with a bound depending only on $\left\|\left(u_{0}, v_{0}\right)\right\|_{1,2}$.

Proof. Part (1) follows easily from the time invariance of the functional

$$
\Phi_{3}=b_{2}\|u(t)\|_{2}^{2}+b_{1}\|v(t)\|_{2}^{2}=b_{2}\left\|u_{0}\right\|_{2}^{2}+b_{1}\left\|v_{0}\right\|_{2}^{2} .
$$

For (2), use is made of the functional $\Phi_{4}$ defined in (1.5). Using the time-invariance of $\Phi_{4}$ together with straightforward estimates leads to the inequality

$$
\begin{aligned}
b_{2}\left\|u_{x}\right\|_{2}^{2}+\left\|v_{x}\right\|_{2}^{2} \leqq & C\left(\left\|u_{0}\right\|_{1,2},\left\|v_{0}\right\|_{1,2}\right)+C_{1}\left(\left\|u_{0}\right\|_{2},\left\|v_{0}\right\|_{2}\right)\left(\|u\|_{\infty}+\|v\|_{\infty}\right) \\
& +2 b_{2}\left|a_{3}\right|\left\|u_{x}\right\|_{2}\left\|v_{x}\right\|_{2} .
\end{aligned}
$$

Using the elementary bound $\|f\|_{\infty} \leqq\|f\|_{2}^{1 / 2}\left\|f_{x}\right\|_{2}^{1 / 2}$, one finds that for $\varepsilon<1$,

$$
\begin{aligned}
b_{2}\left\|u_{x}\right\|_{2}^{2}+\left\|v_{x}\right\|_{2}^{2} \leqq & C+C_{1}\left(\left\|u_{x}\right\|_{2}^{1 / 2}+\left\|v_{x}\right\|_{2}^{1 / 2}\right)+2 b_{2}\left|a_{3}\right|\left\|u_{x}\right\|_{2}\left\|v_{x}\right\|_{2} \\
\leqq & C+C_{1}\left(\left\|u_{x}\right\|_{2}^{1 / 2}+\left\|v_{x}\right\|_{2}^{1 / 2}\right)+2 \sqrt{b_{2}(1-\varepsilon)} \sqrt{\frac{b_{2}}{1-\varepsilon}}\left|a_{3}\right|\left\|u_{x}\right\|_{2}\left\|v_{x}\right\|_{2} \\
\leqq & C+C_{1}\left(\left\|u_{x}\right\|_{2}^{1 / 2}+\left\|v_{x}\right\|_{2}^{1 / 2}\right) \\
& +\left(b_{2}(1-\varepsilon)\left\|u_{x}\right\|_{2}^{2}+\frac{b_{2} a_{3}^{2}}{1-\varepsilon}\left\|v_{x}\right\|_{2}^{2}\right) .
\end{aligned}
$$

The last inequality follows from an application of Young's inequality. It therefore transpires that

$$
b_{2} \varepsilon\left\|u_{x}\right\|_{2}^{2}+\left(1-\frac{b_{2} a_{3}^{2}}{1-\varepsilon}\right)\left\|v_{x}\right\|_{2}^{2} \leqq C+C_{1}\left(\left\|u_{x}\right\|_{2}^{1 / 2}+\left\|v_{x}\right\|_{2}^{1 / 2}\right) .
$$

Note that if $1-b_{2} a_{3}^{2}>0$, then for small, positive $\varepsilon$ we have that $1-\frac{b_{2} a_{3}^{2}}{1-\varepsilon}>0$. Hence, another application of Young's inequality yields the desired result provided $1-b_{2} a_{3}^{2}>0$.

Remark 3.1. The a priori estimates provided in this section would allows us to conclude that the local solutions of $(1.1)$ in $H^{1}(\mathbb{R}) \times H^{1}(\mathbb{R})$ (the existence of which will be shown in Sect. 5) can be globally continued for all $t \geqq 0$ provided $\left|a_{3}\right|<$ $1 / \sqrt{b_{2}}$. 


\section{Linear Estimates}

In this section, the aforementioned estimates established by Kenig, Ponce and Vega for solutions of the linear $\mathrm{KdV}$ equation are extended to solutions of the linear system

$$
\left\{\begin{array}{l}
u_{t}+u_{x x x}+a_{3} v_{x x x}=0 \\
v_{t}+\frac{1}{b_{1}} v_{x x x}+\frac{a_{3} b_{2}}{b_{1}} u_{x x x}=0
\end{array}\right.
$$

where it is assumed that

$$
\alpha_{ \pm}=\frac{1}{2}\left(1+\frac{1}{b_{1}} \pm \sqrt{\left(1-\frac{1}{b_{1}}\right)^{2}+4 \frac{b_{2} a_{3}^{2}}{b_{1}}}\right) \neq 0 .
$$

Without loss of generality we can suppose that $a_{3} \neq 0$, for otherwise the result follows from the previous theory of Kenig et al. For $\alpha_{ \pm}$to be nonzero, it is sufficient to assume that $\sqrt{b_{2}} a_{3} \neq \pm 1$, an assumption weaker than that imposed in Sect. 3 (the latter condition is equivalent to assuming $\alpha_{ \pm}$to be positive). Now let

$$
\lambda=\sqrt{\left(1-\frac{1}{b_{1}}\right)^{2}+4 \frac{b_{2} a_{3}^{2}}{b_{1}}},
$$

and note that by assumption $\lambda$ is positive. Introduce the new dependent variables $w_{1}$ and $w_{2}$ defined as

$$
\begin{aligned}
& w_{1}=\frac{1}{2}\left(1-\frac{1-b_{1}}{\lambda b_{1}}\right) u+\frac{a_{3}}{\lambda} v, \\
& w_{2}=\frac{1}{2}\left(1+\frac{1-b_{2}}{\lambda b_{2}}\right) u-\frac{a_{3}}{\lambda} v .
\end{aligned}
$$

In these variables the system (4.1) can be written in the equivalent, diagonal form

$$
\left\{\begin{array}{l}
w_{1 t}+\alpha_{+} w_{1 x x x}=0 \\
w_{2 t}+\alpha_{-} w_{2 x x x}=0
\end{array}\right.
$$

Since $\alpha_{+}$and $\alpha_{-}$are non-zero, the decoupled, non-degenerate system (4.2) is easily analyzed using the existing theory, and this analysis leads to the desired results for the linear system (4.1).

First, we recall the sharp version of Kato's local smoothing theory for solutions of the K-dV equation (Kenig et al. 1991a, Theorem 4.1).

Theorem 4.1. Let $\left(w_{1}, w_{2}\right)$ be a solution pair of the system (4.2) corresponding to the initial data $\left(w_{10}, w_{20}\right) \in L^{2}(\mathbb{R}) \times L^{2}(\mathbb{R})$. Then there are constants $c_{1}$ and $c_{2}$ such that for any $x \in \mathbb{R}$,

$$
\left(\int_{-\infty}^{\infty}\left|\partial_{x} w_{j}(x, t)\right|^{2} d t\right)^{1 / 2} \equiv c_{j}\left\|w_{0 j}\right\|
$$

for $j=1,2$. 
Next the reader is reminded of the following estimate related to the boundedness of an associated maximal function.

Theorem 4.2. For $j=1,2$ and $w_{j}$ as above, we have that

$$
\left(\int_{-\infty}^{\infty} \sup _{-T, T]}\left|w_{j}(x, t)\right|^{2} d x\right)^{1 / 2} \leqq c_{j}(1+T)^{\rho}\left\|w_{0 j}\right\|_{s, 2}
$$

for any $\rho$ and $s$ which are both larger than $3 / 4$.

Vega (1987) (see also Kenig and Ruiz 1983) showed that this estimate is sharp in the sense that (4.4) does not always hold for $s<3 / 4$.

By interpolation between the estimates (4.3) and (4.4), one obtains immediately the following result which will be used in the next section.

Corollary 4.3. For $j=1,2$ and $w_{j}$ as above, we have that

$$
\left(\int_{-\infty}^{\infty} \int_{-T}^{T}\left|\partial_{x} w_{j}(x, t)\right|^{4} d t d x\right)^{1 / 4} \leqq c_{j}(1+T)^{\gamma}\left\|w_{0 j}\right\|_{l, 2}
$$

for any $\gamma>3 / 8$ and $l>7 / 8$.

Finally, we will make use of a version of the global smoothing effect of Strichartz type (cf. Kenig et al. 1989, Lemma 2.4).

Theorem 4.4. For $w_{1}$ and $w_{2}$ as in Theorem 4.1 and for any $(\theta, \beta) \in[0,1] \times\left[0, \frac{1}{2}\right]$, it follows that

$$
\left(\int_{-\infty}^{\infty}\left\|D^{\theta \beta / 2} w_{j}(\cdot, t)\right\|_{p}^{q} d t\right)^{1 / q} \leqq c_{j}\left\|w_{0 j}\right\|_{2},
$$

for $j=1,2$, where $(q, p)=(6 / \theta(\beta+1), 2 /(1-\theta))$.

Returning to the original problem (4.1), and introducing the notation

$$
\begin{aligned}
W(t)\left(u_{0}, v_{0}\right)(x) & =(u, v)(x, t) \\
& =\left(w_{1}+w_{2}, \frac{1}{2 a_{3}}\left(\frac{1}{b_{1}}-1+\lambda\right) w_{1}-\frac{1}{2 a_{3}}\left(1-\frac{1}{b_{1}}+\lambda\right) w_{2}\right)(x, t)
\end{aligned}
$$

it is easy to see that $u$ and $v$ satisfy estimates analogous to those presented above for $w_{1}$ and $w_{2}$. This remark leads directly to the final result of this section.

Theorem 4.5. The solution of the initial-value problem (4.1) corresponding to initial data $\left(u_{0}, v_{0}\right)$, namely

$$
W(t)\left(u_{0}, v_{0}\right)(x)=(u, v)(x, t)
$$

satisfies the following estimates:

$$
\begin{gathered}
\sup _{x}\left(\int_{-\infty}^{\infty}\left|\partial_{x} W(t)\left(u_{0}, v_{0}\right)(x)\right|^{2} d t\right)^{1 / 2} \leqq C\left\|\left(u_{0}, v_{0}\right)\right\|_{2}, \\
\left(\int_{-\infty}^{\infty} \sup _{-T, T]}\left|W(t)\left(u_{0}, v_{0}\right)(x)\right|^{2} d x\right)^{1 / 2} \leqq C(1+T)^{\rho}\left\|\left(u_{0}, v_{0}\right)\right\|_{s, 2}, \\
\left(\int_{-\infty}^{\infty} \int_{-T}^{T}\left|\partial_{x} W(t)\left(u_{0}, v_{0}\right)(x)\right|^{4} d t d x\right)^{1 / 4} \leqq C(1+T)^{\gamma}\left\|\left(u_{0}, v_{0}\right)\right\|_{l, 2},
\end{gathered}
$$


and

$$
\left(\int_{-\infty}^{\infty}\left\|D^{\theta \beta / 2} W(t)\left(u_{0}, v_{0}\right)(x)\right\|_{p}^{q} d t\right)^{1 / q} \leqq C\left\|\left(u_{0}, v_{0}\right)\right\|_{2},
$$

where $\rho>3 / 4, s>3 / 4, \gamma>3 / 8, l>7 / 8$ and $(\theta, \beta) \in[0,1] \times[0,1 / 2]$ with $(q, p)=$ $(6 / \theta(\beta+1), 2 /(1-\theta))$.

\section{Global Well-Posedness in $H^{s}(\mathbb{R}) \times H^{s}(\mathbb{R}), s \geqq 1$}

In this section, the results obtained in Sect. 2 concerning the local well-posedness of the initial-value problem for the system

$$
\left\{\begin{array}{l}
u_{t}+u u_{x}+u_{x x x}+a_{3} v_{x x x}+a_{1} v v_{x}+a_{2}(u v)_{x}=0 \\
b_{1} v_{t}+v v_{x}+v_{x x x}+b_{2} a_{3} u_{x x x}+b_{2} a_{2} u u_{x}+b_{2} a_{1}(u v)_{x}=0
\end{array}\right.
$$

will be improved. Temporarily, the system (5.1) will be abbreviated as

$$
\left\{\begin{array}{l}
u_{t}+u_{x x x}+a_{3} v_{x x x}+f_{1}\left(u, u_{x}, v, v_{x}\right)=0, \\
v_{t}+\frac{1}{b_{1}} v_{x x x}+\frac{b_{2} a_{3}}{b_{1}} u_{x x x}+f_{2}\left(u, u_{x}, v, v_{x}\right)=0
\end{array}\right.
$$

with $(u, v)(x, 0)=\left(u_{0}, v_{0}\right)(x)$.

Fixing $\left(u_{0}, v_{0}\right) \in H^{1}(\mathbb{R}) \times H^{1}(\mathbb{R})$, consider the initial-value problem for the system (5.2) with initial data $\left(u_{0}^{\varepsilon}, v_{0}^{\varepsilon}\right)=\left(\rho_{\varepsilon} * u_{0}, \rho_{\varepsilon} * v_{0}\right) \in H^{\infty}(\mathbb{R}) \times H^{\infty}(\mathbb{R})$, where $\rho(\cdot) \in \mathscr{S}(\mathbb{R}), \rho \geqq 0, \int_{-\infty}^{\infty} \rho(x) d x=1$ and $\rho_{\varepsilon}(\cdot)=\varepsilon^{-1} \rho\left(\begin{array}{l}\cdot \\ \bar{\varepsilon}\end{array}\right)$. Notice that we do not ask for the moments of $\rho(\cdot)$ to vanish as in Bona and Smith (1975).

We denote by $\left(u^{\varepsilon}, v^{\varepsilon}\right)(x, t)$ the corresponding solution of this problem, defined on the time interval $\left[0, T_{\varepsilon}\right]$ (with the possibility that $T_{\varepsilon} \rightarrow 0$ as $\varepsilon \rightarrow 0$ ) provided by Theorem 2.1. The first goal is to obtain an a priori estimate for the interval $[0, T]$ of existence of the solution $\left(u^{\varepsilon}(\cdot), v^{\varepsilon}(\cdot)\right)$ of (5.2) showing that $T$ is independent of $\varepsilon$ whenever $\left(u_{0}, v_{0}\right) \in H^{1}(\mathbb{R}) \times H^{1}(\mathbb{R})$.

Based on the properties put forth in Sect. 4, and following an argument similar to that given by Kenig et al. (1991b) we shall prove the following result.

Proposition 5.1. With the above notation, there exists $T^{*}=T^{*}\left(\left\|\left(u_{0}, v_{0}\right)\right\|_{1,2}\right)>0$ and $\left.M=M\left(\| u_{0}, v_{0}\right) \|_{1,2}\right)>0$ such that for all $\varepsilon>0$, the solution $\left(u^{\varepsilon}, v^{\varepsilon}\right)(\cdot, t)$ can be extended to the time interval $\left[0, T^{*}\right]$ where it satisfies the following:

$$
\begin{gathered}
\left(u^{\varepsilon}, v^{\varepsilon}\right) \in C\left(0, T^{*} ; H^{\infty}(\mathbb{R}) \times H^{\infty}(\mathbb{R})\right), \\
\sup _{\left[0, T^{*}\right]}\left\{\left\|u^{\varepsilon}(t)\right\|_{1,2}+\left\|v^{\varepsilon}\right\|_{1,2}\right\} \leqq M, \\
\left(\int_{0}^{T^{*}}\left(\left\|\partial_{x} u^{\varepsilon}(t)\right\|_{\infty}^{6}+\left\|\partial_{x} v^{\varepsilon}(t)\right\|_{\infty}^{6}\right) d t\right)^{1 / 6} \leqq M, \\
\left(\int_{-\infty}^{\infty} \int_{0}^{T^{*}}\left(\left|u_{x}\right|^{4}+\left|v_{x}\right|^{4}\right) d t d x\right)^{1 / 4} \leqq M,
\end{gathered}
$$




$$
\sup _{x}\left(\int_{0}^{T^{*}}\left(\left|\partial_{x}^{2} u(x, t)\right|^{2}+\left|\partial_{x}^{2} v(x, t)\right|^{2}\right) d t\right)^{1 / 2} \leqq M,
$$

and

$$
\left(\int_{-\infty}^{\infty}\left(\sup _{\left[0, T^{*}\right]}|u(x, t)|^{2}+\sup _{\left[0, T^{*}\right]}|v(x, t)|^{2}\right) d x\right)^{1 / 2} \leqq M .
$$

Remark 5.1. The results of this proposition still hold if one only assumes that $\left(u_{0}, v_{0}\right) \in H^{s}(\mathbb{R}) \times H^{s}(\mathbb{R})$ with $s>3 / 4$ (with $s$ and $s+1$ replacing 1 and 2 in (5.4) and (5.7) respectively). However, for simplicity of the exposition, consideration is given to the case $s=1$ for which one only encounters derivatives of integer order.

Proof. Using Duhamel's formula, the solution $\left(u^{\varepsilon}, v^{\varepsilon}\right)$ can be written in the form

$$
\left(u^{\varepsilon}, v^{\varepsilon}\right)(\cdot, t)=W(t)\left(u_{0}, v_{0}\right)+\int_{0}^{t} W(t-\tau)\left(f_{1}, f_{2}\right)(\cdot, \tau) d \tau,
$$

where $W(\cdot)$ and $\left(f_{1}, f_{2}\right)$ were defined in (4.7) and (5.2) respectively.

Since $W(t)\left(u_{0}, v_{0}\right)$ denotes the solutions of the linear system $(4.1)$, it is easy to check that

$$
\left\|W(t)\left(u_{0}^{\varepsilon}, v_{0}^{\varepsilon}\right)\right\|_{2} \leqq C\left\|\left(v_{0}^{\varepsilon}, v_{0}^{\varepsilon}\right)\right\|_{2},
$$

where $C$ depends only on $b_{1}$ and $b_{2}$.

Combining (5.9) with Hölder and Sobolev inequalities, and Fubini's theorem shows that for any $T \leqq T_{\varepsilon} \leqq 1$,

$$
\begin{aligned}
\sup _{[0, T]}\|(u, v)(t)\|_{1,2} \leqq & C\left\|\left(u_{0}^{\varepsilon}, v_{0}^{\varepsilon}\right)\right\|_{1,2}+C \int_{0}^{T}\left\|\left(f_{1}, f_{2}\right)(t)\right\|_{2} d t+C \int_{0}^{T}\left\|\left(f_{1 x}, f_{2 x}\right)(t)\right\|_{2} d t \\
\leqq & C\left\|\left(u_{0}, v_{0}\right)\right\|_{1,2}+C \int_{0}^{T}\left(\left\|u u_{x}\right\|_{2}+\left\|v u_{x}\right\|_{2}+\left\|u v_{x}\right\|_{2}+\left\|v v_{x}\right\|_{2}\right) d t \\
& +C T^{1 / 2}\left(\int _ { 0 } ^ { T } \int _ { - \infty } ^ { \infty } \left(\left|u u_{x x}\right|^{2}+\left|u_{x}\right|^{4}+\left|v u_{x x}\right|^{2}+\left|u_{x} v_{x}\right|^{2}\right.\right. \\
& \left.\left.+\left|u v_{x x}\right|^{2}+\left|v_{x}\right|^{4}+\left|v v_{x x}\right|^{2}\right) d x d t\right)^{1 / 2} \\
\leqq & C\left\|\left(u_{0}, v_{0}\right)\right\|_{1,2}+C T\left(\sup _{[0, T]}\|(u, v)(t)\|_{1,2}\right)^{2} \\
& +C T^{1 / 2}\left(\int _ { - \infty } ^ { \infty } \int _ { 0 } ^ { T } \left(\left|u u_{x x}\right|^{2}+\left|u_{x}\right|^{4}+\left|v u_{x x}\right|^{2}\right.\right. \\
& \left.\left.+\left|u_{x} v_{x}\right|^{2}+\left|u v_{x x}\right|^{2}+\left|v_{x}\right|^{4}+\left|v v_{x x}\right|^{2}\right) d t d x\right)^{1 / 2} \\
\leqq & C\left\|\left(u_{0}, v_{0}\right)\right\|_{1,2}+C T^{1 / 2}\left(\sup _{[0, T]}\|(u, v)(t)\|_{1,2}\right)^{2}
\end{aligned}
$$




$$
\begin{aligned}
& +C T^{1 / 2}\left\{\left(\int_{-\infty}^{\infty}\left(\sup _{[0, T]}|u|^{2}+\sup _{[0, T]}|v|^{2}\right) d x\right)^{1 / 2} \times\right. \\
& \left(\sup _{x} \int_{0}^{T}\left(\left|u_{x x}\right|^{2}+\left|v_{x x}\right|^{2}\right) d t\right)^{1 / 2} \\
& \left.+\left(\int_{-\infty}^{\infty} \int_{0}^{T}\left(\left|u_{x}\right|^{4}+\left|v_{x}\right|^{4}\right) d t d x\right)^{1 / 2}\right\} \equiv D,
\end{aligned}
$$

where here and subsequently the subscript $\varepsilon$ has been suppressed. Next using the estimates $(4.11)$ with $(\theta, \beta)=(1,0)$, it follows from $(5.10)$ that for any $T \leqq T_{\varepsilon}$,

$$
\left(\int_{0}^{T}\left\|\partial_{x} u^{\varepsilon}(t)\right\|_{\infty}^{6}+\left\|\partial_{x} v^{\varepsilon}(t)\right\|_{\infty}^{6} d t\right)^{1 / 6} \leqq C\left\|\left(u_{0}^{\varepsilon}, v_{0}^{\varepsilon}\right)\right\|_{1,2}+C \int_{0}^{T}\left\|\left(f_{1 x}, f_{2 x}\right)(t)\right\|_{2} d t \leqq D .
$$

The same argument and the estimates (4.8)-(4.10) show that for $T<T_{\varepsilon} \leqq 1$,

$$
\begin{gathered}
\sup _{x}\left(\int_{0}^{T}\left(\left|u_{x x}\right|^{2}+\left|v_{x x}\right|^{2}\right) d t\right)^{1 / 2} \leqq D, \\
\left(\int_{-\infty}^{\infty}\left(\sup _{[0, T]}|u|^{2}+\sup _{[0, T]}|v|^{2}\right) d x\right)^{1 / 2} \leqq D,
\end{gathered}
$$

and

$$
\left(\int_{-\infty}^{\infty} \int_{0}^{T}\left(\left|u_{x}\right|^{4}+\left|v_{x}\right|^{4}\right) d t d x\right)^{1 / 4} \leqq D
$$

Introducing the notation

$$
\begin{aligned}
X(T)= & \max \left\{\sup _{[0, T]}\|(u, v)(t)\|_{1,2} ; \sup _{x}\left(\int_{0}^{T}\left(\left|u_{x x}\right|^{2}+\left|v_{x x}\right|^{2}\right) d t\right)^{1 / 2} ;\right. \\
& \left(\int_{-\infty}^{\infty}\left(\sup _{[0, T]}|u|^{2}+\sup _{[0, T]}|v|^{2}\right) d x\right)^{1 / 2} ;\left(\int_{-\infty}^{\infty} \int_{0}^{T}\left(\left|u_{x}\right|^{4}+\left|v_{x}\right|^{4}\right) d x d t\right)^{1 / 4} ; \\
& \left.\left(\int_{0}^{T}\left(\left\|u_{x}(t)\right\|_{\infty}^{6}+\left\|v_{x}(t)\right\|_{\infty}^{6}\right) d t\right)^{1 / 6}\right\}
\end{aligned}
$$

(note that $X(T)$ is a non-decreasing function of $T$ as long as the solution $\left(u^{\varepsilon}, v^{\varepsilon}\right)$ remains in the space defined in (5.3)), it is inferred from (5.10)-(5.14) that

$$
X(T) \leqq C\left\|\left(u_{0}, v_{0}\right)\right\|_{1,2}+C T^{1 / 2}(X(T))^{2}
$$

with $T \leqq T_{\varepsilon} \leqq 1$. Now define $T^{*}=\min \left\{1 ; T_{0}\right\}$, where $T_{0}$ is given by the identity

$$
X\left(T_{0}\right)=2 C\left\|\left(u_{0}, v_{0}\right)\right\|_{1,2}=M .
$$

Thus, from (5.16), it is found that

$$
T_{0} \geqq\left(2 C^{2}\left\|\left(u_{0}, v_{0}\right)\right\|_{1,2}\right)^{-2} .
$$

Notice that both estimates (5.17) and (5.18) do not depend on the value of $\varepsilon$. 
If $T_{\varepsilon}<T^{*}$, we obtain (5.17) with $T_{\varepsilon}$ replacing $T_{0}$. Combining this with the energy estimate (2.5), the definition of $X(\cdot)$ and Hölder's inequality yields a bound which allows one to reapply the local existence theorem (see Theorem 2.1 and Lemma 2.3 ) to extend the solution to the time interval $\left[0, T^{*}\right]$ where it satisfies (5.16).

A small modification in the proof allows the removal of the condition $T^{*} \leqq 1$ and thereby proves that $\left.T^{*}=T^{*}\left(\| u_{0}, v_{0}\right) \|_{1,2}\right)$ tends to infinity as $\left\|\left(u_{0}, v_{0}\right)\right\|_{1,2}$ tends to zero. However, this point will not be considered here as our goal is to establish global well-posedness (i.e. $T^{*}=+\infty$ for any $\left(u_{0}, v_{0}\right) \in H^{1}(\mathbb{R}) \times H^{1}(\mathbb{R})$ ).

Next, we shall prove that the net $\left\{\left(u^{\varepsilon}, v^{\varepsilon}\right)\right\} \in C\left(0, T ; H^{\infty}(\mathbb{R}) \times H^{\infty}(\mathbb{R})\right)$ converges in $C\left(0, T ; H^{1}(\mathbb{R}) \times H^{1}(\mathbb{R})\right)$ for some $T<T^{*}$.

Proposition 5.2. For some $T<T^{*}$, the family $\left\{\left(u^{\varepsilon}, v^{\varepsilon}\right)\right\}_{\varepsilon>0}$ converges on the interval $[0, T]$ in the norms appearing on the left-hand sides of (5.4)-(5.8) to a strong solution $(u, v) \in C\left(0, T ; H^{1}(\mathbb{R}) \times H^{1}(\mathbb{R})\right)$ of the system $(5.1)$.

Proof. The argument is similar to that given in the proof of the previous proposition, so, a sketch will suffice. For $\varepsilon>\varepsilon^{\prime}>0$, define $\left(z_{1}, z_{2}\right)=\left(u^{\varepsilon}-u^{\varepsilon^{\prime}}, v^{\varepsilon}-v^{\varepsilon^{\prime}}\right)$. This pair satisfies

$$
\begin{array}{r}
z_{1 t}+z_{1 x x x}+a_{3} z_{2 x x x}+u_{x}^{\varepsilon} z_{1}-u^{\varepsilon^{\prime}} z_{1 x}+a_{1}\left(v_{x}^{\varepsilon} z_{2}-v^{\varepsilon^{\prime}} z_{2 x}\right)+a_{2}\left(v^{\varepsilon} z_{1}-u^{\varepsilon^{\prime}} z_{2}\right)_{x}=0, \\
b_{1} z_{2 t}+z_{2 x x x}+b_{2} a_{3} z_{1 x x x}+v_{x}^{\varepsilon} z_{2}-v^{\varepsilon^{\prime}} z_{2 x}+b_{2} a_{2}\left(u_{x}^{\varepsilon} z_{1}-u^{\varepsilon^{\prime}} z_{1 x}\right)+b_{2} a_{1}\left(v^{\varepsilon} z_{1}-u^{\varepsilon^{\prime}} z_{2}\right)_{x}=0,
\end{array}
$$

with initial data $\left(z_{10}, z_{20}\right)=\left(u_{0}^{\varepsilon}-u_{0}^{\varepsilon^{\prime}}, v_{0}^{\varepsilon}-v_{0}^{\varepsilon^{\prime}}\right)$.

As in the previous proof, define $Y(T)$ in analogy with $X(T)$ in (5.15), where $\left(z_{1}, z_{2}\right)$ replaces $(u, v)$. Following the same argument exposed in (5.8)-(5.14), it is discovered that for $T<T^{*}$,

$$
Y(T) \leqq C\left\|\left(z_{10}, z_{20}\right)\right\|_{1,2}+C T^{1 / 2} M Y(T),
$$

where the constant $M$ was defined in (5.17). Hence, fixing $T \leqq T^{*}$ such that

it is concluded that

$$
C T^{1 / 2} M=\frac{1}{2}
$$

$$
Y(T)=o(1)
$$

when $\varepsilon>\varepsilon^{\prime}>0$ tend to zero. This completes the proof.

Combining Proposition 5.1 and 5.2 leads to the local well-posedness result advertised earlier.

Theorem 5.3. The following points are valid concerning the initial-value problem for the system (5.1).

(i) For any $\left(u_{0}, v_{0}\right) \in H^{1}(\mathbb{R}) \times H^{1}(\mathbb{R})$, there exists $T=T\left(\left\|\left(u_{0}, v_{0}\right)\right\|_{1,2}\right)>0$ and $a$ unique strong solution $(u, v)$ of the system $(5.1)$ with $\left(u_{0}, v_{0}\right)$ as initial data such that

$$
\begin{gathered}
(u, v) \in C\left(0, T ; H^{1}(\mathbb{R}) \times H^{1}(\mathbb{R})\right), \\
\left(u_{x}, v_{x}\right) \in L^{6}\left(0, T ; L^{\infty}(\mathbb{R}) \times L^{\infty}(\mathbb{R})\right),
\end{gathered}
$$

and

$$
\sup _{x}\left(\int_{0}^{T}\left(\left|\partial_{x}^{2} u(x, t)\right|^{2}+\left|\partial_{x}^{2} v(x, t)\right|^{2}\right) d t\right)^{1 / 2}<\infty
$$


In particular, (5.22) guarantees that

$$
(u, v) \in L^{2}\left(0, T ; H_{\mathrm{loc}}^{2}(\mathbb{R}) \times H_{\mathrm{loc}}^{2}(\mathbb{R})\right) .
$$

(ii) Moreover, the solution $(u, v)$ in the space given in (5.20) and those defined by the norm used in (5.21)-(5.22) depends continuously on the data in $H^{1}(\mathbb{R}) \times H^{1}(\mathbb{R})$.

(iii) If in addition $\left(u_{0}, v_{0}\right) \in H^{s}(\mathbb{R}) \times H^{s}(\mathbb{R})$ with $s>1$, then the solution $(u, v)$ belongs to $C\left(0, T ; H^{s}(\mathbb{R}) \times H^{s}(\mathbb{R})\right)$.

Proof. The proofs of the parts (ii)-(iii) are similar to that provided in detail for part (i), and therefore they will be omitted here. Uniqueness is immediate since

$$
\begin{aligned}
\int_{0}^{T}\left(\left\|u_{x}(t)\right\|_{\infty}+\left\|v_{x}(t)\right\|_{\infty}\right) d t & \leqq T^{5 / 6}\left(\int_{0}^{T}\left(\left\|u_{x}(t)\right\|_{\infty}+\left\|v_{x}(t)\right\|_{\infty}\right)^{6} d t\right)^{1 / 6} \\
& \leqq c T^{5 / 6}\left(\int_{0}^{T}\left(\left\|u_{x}(t)\right\|_{\infty}^{6}+\left\|v_{x}(t)\right\|_{\infty}^{6}\right) d t\right)^{1 / 6} \\
& \leqq c T^{5 / 6} X(T) .
\end{aligned}
$$

Theorem 5.4. (Global Well-Posedness). If $\left|a_{3}\right|<1 / \sqrt{b_{2}}$, then the results in Theorem 5.3 are true with $T$ arbitrarily large.

Proof. Theorem 5.4 follows by combining Theorem 5.3 with Lemma 3.1.

Remark 5.2. (1) The linear estimates in Sect. 4 depend on the fact that the eigenvalues $\left\{\alpha_{ \pm}\right\}$of the coefficient matrix of the dispersive terms in (4.1) are both nonzero. If either of these eigenvalues is zero, then the corresponding equation in (4.2) is hyperbolic and hence no smoothing effect can be derived from it. As a consequence, we would not have the local well-posedness result (Theorem 5.3).

(2) It should be noted that as long as $b_{1}$ is positive and finite (regardless of its magnitude), the existence of global solutions in $H^{1}(\mathbb{R}) \times H^{1}(\mathbb{R})$ is assured provided the condition $\left|a_{3}\right|<1 / \sqrt{b_{2}}$ is satisfied. Indeed this condition is satisfied by the examples given by Gear and Grimshaw (1984) wherein (i) $a_{3}=0.139389, b_{1}=$ $2.267029, b_{2}=21.513946$ and (ii) $a_{3}=0.5, b_{1}=b_{2}=2$. For (i) $1 / \sqrt{b_{2}}=0.2155956$ and for (ii) $1 / \sqrt{b_{2}}=0.7071068$.

\section{Local Well-Posedness in $L^{2}$ and Dispersive Blow-up}

In this section use is made of Kato's original local smoothing ideas to obtain existence of solutions to the initial-value problem for (5.1) corresponding to data which lies only in $L^{2}(\mathbb{R}) \times L^{2}(\mathbb{R})$. This result in turn will be used in the analysis of a certain type of singularity formation termed dispersive blow-up by Bona and Saut (1991a).

Let $\left(u_{0}, v_{0}\right) \in L^{2}(\mathbb{R}) \times L^{2}(\mathbb{R})$ be a given pair of initial data for (5.1) and suppose that $\left(u_{0, n}, v_{0, n}\right) \in H^{\mathbf{3}}(\mathbb{R}) \times H^{3}(\mathbb{R})$ are smoother data which converge to $\left(u_{0}, v_{0}\right)$ in $L^{2}(\mathbb{R}) \times L^{2}(\mathbb{R})$ as $n$ tends to infinity. Presuming that $\left|a_{3}\right|<1 / \sqrt{b_{2}}$, Theorem 5.4 assures that the system (5.1) has a unique solution pair $\left(u_{n}, v_{n}\right) \in C\left(0, \infty ; H^{3}(\mathbb{R})\right) \times$ $C\left(0, \infty ; H^{3}(\mathbb{R})\right)$ corresponding to the initial data $\left(u_{0, n}, v_{0, n}\right)$. 
Dropping the subscript $n$, and writing $(u, v)$ for $\left(u_{n}, v_{n}\right)$, we proceed to derive bounds on $\left(u_{n}, v_{n}\right)$. Let $p$ be a $C^{\infty}$ real-valued function which is bounded on $\mathbb{R}$ along with its first few derivatives, and which is such that $p_{x}>0$ for all $x \in \mathbb{R}$. Multiply the first equation in (5.1) by $b_{2} p u$ and the second equation in (5.1) by $p v$ and integrate the results with respect to $x$ over $\mathbb{R}$. After several, justifiable integrations by parts, there appears the relation

$$
\begin{aligned}
& \frac{b_{2}}{2} \frac{d}{d t} \int_{-\infty}^{\infty} p u^{2} d x+\frac{b_{1}}{2} \frac{d}{d t} \int_{-\infty}^{\infty} p v^{2}+\frac{3 b_{2}}{2} \int_{-\infty}^{\infty} p_{x} u_{x}^{2} d x+\frac{3}{2} \int_{-\infty}^{\infty} p_{x} v_{x}^{2} d x \\
& =\frac{b_{2}}{2} \int_{-\infty}^{\infty} p_{x x x} u^{2} d x+\frac{1}{2} \int_{-\infty}^{\infty} p_{x x x} v^{2} d x-3 b_{2} a_{3} \int_{-\infty}^{\infty} p_{x} u_{x} v_{x} d x \\
& \quad+b_{2} a_{3} \int_{-\infty}^{\infty} p_{x x x} u v d x+b_{2} a_{1} \int_{-\infty}^{\infty} p_{x} u v^{2} d x+b_{2} a_{2} \int_{-\infty}^{\infty} p_{x} u^{2} v d x \\
& \quad+\frac{1}{3} \int_{-\infty}^{\infty} p_{x} v^{3} d x+\frac{b_{2}}{3} \int_{-\infty}^{\infty} p_{x} u^{3} d x .
\end{aligned}
$$

If Eq. (6.1) is integrated in time over the interval $[0, T]$ and use is made of the invariance of the functional $\Phi_{3}$, and thus the boundedness of $\|u(\cdot, t)\|_{2}$ and $\|v(\cdot, t)\|_{2}$, independently of $t \geqq 0$ and $n=1,2, \ldots$, and the properties of $p$, then one obtains that

$\frac{3}{2} \int_{0}^{T} \int_{-\infty}^{\infty}\left(b_{2} p_{x} u_{x}^{2}+p_{x} v_{x}^{2}\right) d x d t$

$$
\begin{aligned}
\leqq & C\left(T, p,\left\|u_{0}\right\|_{2},\left\|v_{0}\right\|_{2}\right)+3 b_{2}\left|a_{3}\right| \int_{0}^{T}\left|\int_{-\infty}^{\infty} p_{x} u_{x} v_{x} d x\right| d t+b_{2}\left|a_{1}\right| \int_{0}^{T}\left|\int_{-\infty}^{\infty} p_{x} u v^{2} d x\right| d t \\
& +b_{2}\left|a_{2}\right| \int_{0}^{T}\left|\int_{-\infty}^{\infty} p_{x} u^{2} v d x\right| d t+\frac{b_{2}}{3} \int_{0}^{T}\left|\int_{-\infty}^{\infty} p_{x} u^{3} d x\right| d t+\frac{1}{3} \int_{0}^{T}\left|\int_{-\infty}^{\infty} p_{x} v^{3} d x\right| d t
\end{aligned}
$$

Using again the elementary inequality $\|f\|_{\infty} \leqq\|f\|_{2}^{1 / 2}\left\|f^{\prime}\right\|_{2}^{1 / 2}$, the terms that are cubic in $u, v$ in (6.2) may be estimated as follows:

$$
\begin{aligned}
& \left|\int_{-\infty}^{\infty} p_{x} u^{3} d x\right| \leqq\left(\left\|p_{x} u\right\|_{2}^{1 / 2}\left\|p_{x x} u\right\|_{2}^{1 / 2}+\left\|p_{x} u\right\|_{2}^{1 / 2}\left\|p_{x} u_{x}\right\|_{2}^{1 / 2}\right)\|u\|_{2}^{2} \\
& \left|\int_{-\infty}^{\infty} p_{x} v^{3} d x\right| \leqq\left(\left\|p_{x} v\right\|_{2}^{1 / 2}\left\|p_{x x} v\right\|_{2}^{1 / 2}+\left\|p_{x} v\right\|_{2}^{1 / 2}\left\|p_{x} v_{x}\right\|_{2}^{1 / 2}\right)\|v\|_{2}^{2} \\
& \left|\int_{-\infty}^{\infty} p_{x} u v^{2} d x\right| \leqq\left(\left\|p_{x} u\right\|_{2}^{1 / 2}\left\|p_{x x} u\right\|_{2}^{1 / 2}+\left\|p_{x} u\right\|_{2}^{1 / 2}\left\|p_{x} u_{x}\right\|_{2}^{1 / 2}\right)\|v\|_{2}^{2}, \\
& \left|\int_{-\infty}^{\infty} p_{x} u^{2} v d x\right| \leqq\left(\left\|p_{x} v\right\|_{2}^{1 / 2}\left\|p_{x x} v\right\|_{2}^{1 / 2}+\left\|p_{x} v\right\|_{2}^{1 / 2}\left\|p_{x} v_{x}\right\|_{2}^{1 / 2}\right)\|u\|_{2}^{2}
\end{aligned}
$$

If it is now assumed that $b_{2} a_{3}^{2}<1$, then (6.2), (6.3), the invariance of $\Phi_{3}$ and Young's inequality imply that

$$
\int_{0}^{T} \int_{-\infty}^{\infty} p_{x}\left(u_{x}^{2}+v_{x}^{2}\right) d x d t \leqq C\left(T, p,\left\|u_{0}\right\|_{2},\left\|v_{0}\right\|_{2}\right)
$$


and therefore, by an appropriate choice of the increasing function $p$, that

$$
\int_{0}^{T} \int_{-R}^{R}\left(u_{x}^{2}+v_{x}^{2}\right) d x d t \leqq C\left(T, R,\left\|u_{0}\right\|_{2},\left\|v_{0}\right\|\right)
$$

for all finite, positive values of $T$ and $R$. It is thus concluded from (6.5) and the invariance of $\Phi_{3}$ that the sequences

$$
\left\{u_{n}\right\}_{n=1}^{\infty} \text { and }\left\{v_{n}\right\}_{n=1}^{\infty} \text { are bounded in } L^{\infty}\left(0, T ; L^{2}(\mathbb{R})\right) \cap L^{2}\left(0, T ; H^{1}(-R, R)\right) \text {, }
$$

independently of $n$, for finite values of $R$ and T. Using Eqs. (5.1) satisfied by $\left(u_{n}, v_{n}\right)$, it is then straightforward to conclude that for each $T, R>0$, the sequences

$$
\left\{\partial_{t} u_{n}\right\}_{n=1}^{\infty} \text { and }\left\{\partial_{t} v_{n}\right\}_{n=1}^{\infty} \text { are bounded in } L^{2}\left(0, T ; H^{-2}(-R, R)\right) \text {, }
$$

independently of $n$. It is then standard to use the Aubin-Lions compactness result to pass to the limit in (5.1) as $n$ tends to infinity, so obtaining the following existence theorem.

Theorem 6.1. Assume that $\left|a_{3}\right|<1 / \sqrt{b_{2}}$ and let $\left(u_{0}, v_{0}\right) \in L^{2}(\mathbb{R}) \times L^{2}(\mathbb{R})$. Then the system (5.1) has a solution $(u, v)$ corresponding to the initial data $\left(u_{0}, v_{0}\right)$ such that

$$
u, v \in L^{\infty}\left(0, \infty ; L^{2}(\mathbb{R})\right) \cap L^{2}\left(0, T ; H^{1}(-R, R)\right)
$$

for each $T, R>0$. Any such solution has the property that

$$
u_{t}, v_{t} \in L^{2}\left(0, T ; H_{\mathrm{loc}}^{-2}(\mathbb{R})\right)
$$

and hence

$$
u, v \in C\left(0, T ; H_{\mathrm{loc}}^{-1 / 2}(\mathbb{R})\right) \cap C_{w}\left(0, T ; H_{\mathrm{loc}}^{1}(\mathbb{R})\right) .
$$

The initial values are taken on at least in the sense of the latter space.

Attention is now given to the so-called dispersive blow-up properties which obtain for the Gear-Grimshaw system (5.1) in much the same way as for the generalized Kortweg-de Vries equation. For simplicity, the theory is only developed in the $L^{2}$-context, though the reader will readily appreciate how a theory relative to $H^{k}$ can be carried out along the same lines. Indeed, the theorem stated below encompasses this generalization.

Theorem 6.2. Assume that $\left|a_{3}\right|<1 / \sqrt{b_{2}}$ and let a non-negative integer $k$ and real numbers $T>0, x^{*} \in \mathbb{R}$ and $0<t^{*}<T$ be given. Then there exists initial data $u_{0}, v_{0}$ in $H^{k}(\mathbb{R}) \cap C_{b}^{k}(\mathbb{R}) \cap C^{\infty}(\mathbb{R})$ and a corresponding solution pair $(u, v)$ of (5.1) such that $u, v \in L^{\infty}\left(0, T ; H^{k}(\mathbb{R})\right) \cap L^{2}\left(0, T ; H_{\mathrm{loc}}^{k+1}(\mathbb{R})\right), \partial_{x}^{k} u, \partial_{x}^{k} v$ are both continuous functions of $(x, t)$ in the domain $\mathbb{R} \times(0, T) \backslash\left\{\left(x^{*}, t^{*}\right)\right\}$, and

$$
\lim _{\substack{x \rightarrow x^{*} \\ t \rightarrow t^{*}}}\left|\partial_{x}^{k} u(x, t)\right|=\lim _{\substack{x \rightarrow x^{*} \\ t \rightarrow t^{*}}}\left|\partial_{x}^{k} v(x, t)\right|=+\infty .
$$

Remark 6.3. By $C_{b}^{k}(\mathbb{R})$ we mean the $C^{k}$-functions defined on $\mathbb{R}$ whose derivatives up to order $k$ are uniformly bounded on $\mathbb{R}$. In case $k \geqq 1$ above, the solution pair $(u, v)$ is unique and $u, v$ actually lie in $C\left(0, T ; H^{k}(\mathbb{R})\right)$, as stated already in Theorem 5.3.

Proof. As mentioned above, the proof is sketched here only for the case $k=0$. The line of argument follows very closely that appearing in Bona and Saut (1991a). 
The first step in the proof is to obtain an existence result for solutions of (5.1) in weighted spaces. Consider the special class of weights $w=w_{\sigma}$ which are non-decreasing $C^{\infty}$ functions depending on the positive parameter $\sigma$ such that

$$
w(x)=w_{\sigma}(x)=\left\{\begin{array}{cll}
1 & \text { for } & x<0,
\end{array}\right. \text { and }
$$

The class $H^{k}(\mathbb{R}, w)$ is the class of $H^{k}(\mathbb{R})$-function whose derivatives up to order $k$ are square integrable with respect to the measure $w^{2}(x) d x$. If $k=0$, this space is denoted $L^{2}(\mathbb{R}, w)$.

Proposition 6.4. Assume that $\left|a_{3}\right|<1 / \sqrt{b_{2}}$ and suppose that the initial data $\left(u_{0}, v_{0}\right)$ for the system (5.1) lies in $L^{2}(\mathbb{R}, w) \times L^{2}(\mathbb{R}, w)$. Then there exists a solution pair $(u, v)$ to (5.1) corresponding to $\left(u_{0}, v_{0}\right)$ as in Theorem 6.1 such that for any $T>0$, one has $u, v \in L^{\infty}\left(0, T ; L^{2}(\mathbb{R}, w)\right)$.

Proof. The argument is made as in the proof of Theorem 6.1 by working with initial data which is smooth (e.g. $\left.u_{0, n}, v_{0, n} \in C_{0}^{\infty}(\mathbb{R}), n=1,2, \ldots\right)$ and which converges to $u_{0}, v_{0}$ in $L^{2}(\mathbb{R}, w)$. It is then only required to derive a priori bounds on the associated solution $\left(u_{n}, v_{n}\right), n=1,2, \ldots$, in $L^{2}(\mathbb{R}, w)$ in order that, when the limit is taken as $n$ tends to infinity, the resulting weak solution may be inferred to lie in $L^{\infty}\left(0, T ; L^{2}(\mathbb{R}, w)\right) \times L^{\infty}\left(0, T ; L^{2}(\mathbb{R}, w)\right)$.

To this just mentioned end, define $A=u w$ and $B=v w$ so that $A, B$ satisfy the slightly complicated system of equations

$$
\begin{aligned}
A_{t} & +A_{x x x}+a_{3} B_{x x x}+\left(A+a_{3} B\right)\left(6 \frac{w_{x} w_{x x}}{w^{2}}-6 \frac{w_{x}^{3}}{w^{3}}-\frac{w_{x x x}}{w}\right) \\
& +\left(A_{x}+a_{3} B_{x}\right)\left(6 \frac{w_{x}^{2}}{w^{2}}-3 \frac{w_{x x}}{w}\right)-3 \frac{w_{x}}{w}\left(A_{x x}+a_{3} B_{x x}\right)+a_{1}\left(\frac{1}{w} B B_{x}-\frac{w_{x}}{w^{2}} B^{2}\right) \\
& +a_{2}\left(\frac{1}{w}(A B)_{x}-2 \frac{w_{x}}{w^{2}} A B\right)+\frac{1}{w} A A_{x}-\frac{w_{x}}{w^{2}} A^{2}=0 \\
b_{1} B_{t} & +b_{2} a_{3} A_{x x x}+B_{x x x}+\left(b_{2} a_{3} A+B\right)\left(6 \frac{w_{x} w_{x x}}{w^{2}}-6 \frac{w_{x}^{3}}{w^{3}}-\frac{w_{x x x}}{w}\right) \\
& +\left(b_{2} a_{3} A_{x}+B_{x}\right)\left(6 \frac{w_{x}^{2}}{w^{2}}-3 \frac{w_{x x}}{w}\right)-3 \frac{w_{x}}{w}\left(b_{2} a_{3} A_{x x}+B_{x x}\right) \\
& +\frac{1}{w} B B_{x}-\frac{w_{x}}{w^{2}} B^{2}+\frac{b_{2} a_{2}}{w} A A_{x}-b_{2} a_{2} \frac{w_{x}}{w^{2}} A^{2} \\
& +b_{2} a_{1}\left(\frac{1}{w}(A B)_{x}-2 \frac{w_{x}}{w^{2}} A B\right)=0 .
\end{aligned}
$$

All the coefficients appearing in (6.10) are smooth and bounded, because of the properties of $w$, and hence this system admits a local existence theory along the lines enunciated in Sect. 2. Since for each integer $n$, the initial data $A(x, 0)=w(x) u_{0, n}(x)$ and $B(x, 0)=w(x) v_{0, n}(x)$ lies in $H^{\infty}(\mathbb{R})$, it will follow that the initial-value problems for (6.10) possess unique solutions $(A, B)=\left(A_{n}, B_{n}\right)$, such that 
$A, B \in C\left(0, T ; H^{k}(\mathbb{R})\right)$ for some $T>0$ and any $k$. By uniqueness for the initial-value problem for (5.1), it follows that $A_{n}=w u_{n}$ and $B_{n}=w v_{n}$. In any case, we are thus assured of solutions of the system (6.10) having more than enough regularity and decay at infinity to justify the quest for energy-type estimates upon which we now embark.

Multiply the first Eq. (6.8) by $b_{2} A$ and the second by $B$, integrate the results over $\mathbb{R}$, and integrate by parts to reach the relation

$$
\begin{aligned}
\frac{1}{2} b_{2} & \frac{d}{d t}\|A\|_{2}^{2}+\frac{1}{2} b_{1} \frac{d}{d t}\|B\|_{2}^{2}+3 b_{2} \int_{-\infty}^{\infty} \frac{w_{x}}{w} A_{x}^{2} d x+3 \int_{-\infty}^{\infty} \frac{w_{x}}{w} B_{x}^{2} d x \\
= & 6 b_{2} a_{3} \int_{-\infty}^{\infty} \frac{w_{x}}{w} A_{x} B_{x} d x+3 b_{2} a_{3} \int_{-\infty}^{\infty}\left(\frac{w_{x}}{w}\right)_{x x} A B d x+\int_{-\infty}^{\infty} \theta_{1} A^{2} d x \\
& +\int_{-\infty}^{\infty} \theta_{2} B^{2} d x+\int_{-\infty}^{\infty} \theta_{3} A B d x+\frac{2 b_{2}}{3} \int_{-\infty}^{\infty} \frac{w_{x}}{w^{2}} A^{3} d x+\frac{2}{3} \int_{-\infty}^{\infty} \frac{w_{x}}{w^{2}} B^{3} d x \\
& -a_{1} b_{2} \int_{-\infty}^{\infty} \frac{1}{w}\left[A B B_{x}+(A B)_{x} B\right] d x-a_{2} b_{2} \int_{-\infty}^{\infty} \frac{1}{w}\left[(A B)_{x} A+A A_{x} B\right] d x \\
& +3 a_{1} b_{2} \int_{-\infty}^{\infty} \frac{w_{x}}{w_{2}} A B^{2} d x+3 a_{2} b_{2} \int_{-\infty}^{\infty} \frac{w_{x}}{w^{2}} A^{2} B d x,
\end{aligned}
$$

where, due to the properties of $w, \theta_{1}, \theta_{2}$ and $\theta_{3}$ are smooth functions which are bounded, along with all their derivatives. First notice that

$$
\left|6 b_{2} a_{3} \int_{-\infty}^{\infty} \frac{w_{x}}{w} A_{x} B_{x} d x\right|<\left|a_{3}\right| \sqrt{b_{2}}\left(\int_{-\infty}^{\infty} \frac{w_{x}}{w}\left(b_{2} A_{x}^{2}+B_{x}^{2}\right) d x\right) .
$$

Further integration by parts shows that

$$
-a_{1} b_{2} \int_{-\infty}^{\infty} \frac{1}{w}\left(A B B_{x}+B(A B)_{x}\right) d x=-a_{1} b_{2} \int_{-\infty}^{\infty} \frac{w_{x}}{w^{2}} A B^{2} d x
$$

and similarly

$$
-a_{1} b_{2} \int_{-\infty}^{\infty} \frac{1}{w}\left(A(A B)_{x}+A A_{x} B\right) d x=-a_{2} b_{2} \int_{-\infty}^{\infty} \frac{w_{x}}{w^{2}} A^{2} B d x .
$$

Estimating straightforwardly in (6.11) thus leads to the inequality

$$
\begin{aligned}
& \frac{1}{2} \frac{d}{d t}\left(b_{2}\|A\|_{2}^{2}+b_{1}\|B\|_{2}^{2}\right)+3 \int_{-\infty}^{\infty} \frac{w_{x}}{w}\left(b_{2} A_{x}^{2}+B_{x}^{2}\right) d x \\
& \leqq \\
& c_{1}\|A\|_{2}^{2}+c_{2}\|B\|_{2}^{2}+6 a_{2} b_{3}\left|\int_{-\infty}^{\infty} \frac{w_{x}}{w} A_{x} B_{x} d x\right| \\
& +\frac{2}{3}\left|\int_{-\infty}^{\infty} \frac{w_{x}}{w}\left(b_{2} A^{3}+B^{3}\right) d x\right|+2 b_{2}\left|\int_{-\infty}^{\infty} \frac{w_{x}}{w^{2}}\left(a_{1} A B^{2}+a_{2} A^{2} B\right) d x\right| .
\end{aligned}
$$

Proceeding exactly as in the proof of Theorem 3.1 in Bona and Saut (1991a, the case $p=1$ ), one readily derives that the last two terms on the right-hand side of 
(6.13) are majorized by an expression of the form

$$
C(\eta)\left(\|A\|_{2}^{2}+\|B\|_{2}^{2}\right)+\eta \int_{-\infty}^{\infty} \frac{w_{x}}{w}\left(b_{2} A_{x}^{2}+B_{x}^{2}\right) d x,
$$

where $\eta>0$ is arbitrary and $C(\eta)$ depends inversely upon $\eta$. Combining this with (6.12) and (6.13), using the hypothesis that $\left|a_{3}\right|<1 / \sqrt{b_{2}}$, and choosing $\eta$ small enough, it is adduced that there is a $\delta>0$ such that

$$
\frac{1}{2} \frac{d}{d t}\left(b_{2}\|A\|_{2}^{2}+b_{1}\|B\|_{2}^{2}\right)+\delta \int_{-\infty}^{\infty} \frac{w_{x}}{w}\left(b_{2} A_{x}^{2}+B_{x}^{2}\right) d x \leqq C_{3}\left(\|A\|_{2}^{2}+\|B\|_{2}^{2}\right),
$$

and this in turn leads to a priori bounds on $A$ and $B$ in the space $L^{\infty}\left(0, T ; L^{2}(\mathbb{R})\right) \cap L^{2}\left(0, T ; H_{\mathrm{loc}}^{1}(\mathbb{R})\right)$. In fact, the Gronwall lemma yields bounds on $A$ and $B$ in $L^{\infty}\left(0, T ; L^{2}(\mathbb{R})\right)$ and then, by integrating the last inequality over the temporal interval $[0, T]$ and using the fact that $A(\cdot . T)$ and $B(\cdot, T)$ are bounded in $L^{2}(\mathbb{R})$, it is concluded that

$$
\int_{0}^{T} \int_{-\infty}^{\infty} \frac{w_{x}}{w}\left(b_{2} A_{x}^{2}+B_{x}^{2}\right) d x d t
$$

is bounded, and that the bound only depends upon $T$, the weight function $w$, and the $L^{2}$-norm of the initial data $A_{0}$ and $B_{0}$. Since $b_{2}>0$, it thus follows that for any finite value of $K>1$,

$$
\int_{0}^{T} \int_{1}^{K}\left(A_{x}^{2}+B_{x}^{2}\right) d x d t
$$

is bounded with a bound that again only depends upon $T$, the weight function $w$ and the $L^{2}$-norm of the initial data. By considering spatial translates of the weight function, the desired bounds on $A, B$ in $L^{2}\left(0, T ; H_{10 c}^{1}(\mathbb{R})\right)$ are then easily concluded. In particular, it is seen that for any $T>0$, there is a constant $C=C\left(T, R, \sigma,\left\|A_{0}\right\|_{2},\left\|B_{0}\right\|_{2}\right)$ depending only on $T, R$, the value of $\sigma$ in the definition of $w$, and the $L^{2}$-norm of the initial data for $A$ and $B$ such that

$$
\|A(\cdot, t)\|_{2}+\|B(\cdot, t)\|_{2}+\int_{0}^{T} \int_{-R}^{R}\left(A_{x}^{2}(x, t)+B_{x}^{2}(x, t)\right) d x d t \leqq C
$$

for $0 \leqq t \leqq T$. Thus in the situation at hand, wherein $(A, B)=\left(A_{n}, B_{n}\right)$, but where the initial data $\left(A_{0, n}, B_{0, n}\right)$ remains uniformly bounded in $L^{2}(\mathbb{R}) \times L^{2}(\mathbb{R})$, the entire sequence $\left\{\left(A_{n}, B_{n}\right)\right\}_{n=1}^{\infty}$ is concluded to be bounded in $L^{\infty}\left(0, T ; L^{2}(\mathbb{R})\right) \cap$ $L^{2}\left(0, T ; H_{\mathrm{loc}}^{1}(\mathbb{R})\right)$.

Energy-type estimates may be derived in the same way in $L^{\infty}\left(0, T ; H^{j}(\mathbb{R})\right)$, $j=1,2, \ldots$, since the initial data $A_{0, n}, B_{0, n}$ lies in $H^{\infty}(\mathbb{R})$ (cf. again Bona and Saut 1991a, Theorem 3.1). These bounds may be used to conclude that the local solution guaranteed by Kato's theory is in fact global in time. However, since $\left\{\left(A_{0, n}, B_{0, n}\right)\right\}_{n=1}^{\infty}$ does not remain bounded in $H^{j}(\mathbb{R})$ for $j \geqq 1$, nothing can be concluded about boundedness of the sequence of solutions $\left\{\left(A_{n}, B_{n}\right)\right\}_{n=1}^{\infty}$ in such Sobolev spaces. Of course, these cases come especially to the fore when the $k$ in the statement of the theorem is larger than zero. 
In all events, the bound in (6.14) allows one to pass to the limit as $n$ tends to infinity and so establish the veracity of the proposition.

With Proposition 6.3 in hand, attention is refocussed on the proof of Theorem 6.2. Consider the potential blow-up point $\left(x^{*}, t^{*}\right)$. By a translation of the spatial variable, we may take it that $x^{*}=0$ without loss of generality.

The idea is to choose initial data $\left(u_{0}, v_{0}\right)$ which is such that when the linearized initial-value problem is solved, the solution forms the desired singularity at the point $\left(0, t^{*}\right)$. Then using Duhamel's principle, the solution of the full system is written as the solution of the linearized problem plus an integral term involving the linear solution-semigroup and the nonlinear terms. The first term in the last-mentioned sums forms a singularity at $\left(0, t^{*}\right)$, whilst the second will be shown to be well-behaved, thus leading to the desired conclusion.

First consider the decoupled system (4.2) where, since $\left|a_{3}\right|<1 / \sqrt{b_{2}}$, the eigenvalues $\alpha_{ \pm}$introduced in Sect. 4 are positive. According to the theory developed in Bona and Saut (1991a), if $w_{i}(\cdot, 0)$ is chosen as

$$
w_{i}(x, 0)=\frac{A i\left(-\beta_{i} x\right)}{\left(1+x^{2}\right)^{m}}
$$

for $i=1,2$, where $\frac{3}{16}<m \leqq \frac{1}{4}$, then the solution of (4.2), namely

$$
\begin{aligned}
& w_{1}(x, t)=\frac{1}{\left(\alpha_{+} t\right)^{1 / 3}} \int_{-\infty}^{\infty} A i\left(\frac{x-y}{\left(\alpha_{+} t\right)^{1 / 2}}\right) A i\left(-\beta_{1} y\right)\left(1+y^{2}\right)^{-m} d y, \\
& w_{2}(x, t)=\frac{1}{\left(\alpha_{-} t\right)^{1 / 3}} \int_{-\infty}^{\infty} A i\left(\frac{x-y}{\left(\alpha_{-} t\right)^{1 / 2}}\right) A i\left(-\beta_{2} y\right)\left(1+y^{2}\right)^{-m} d y,
\end{aligned}
$$

has the following properties. First the initial data is such that $w_{i}(\cdot, 0) \in L^{2}(\mathbb{R}) \cap$ $C_{b}(\mathbb{R}) \cap C^{\infty}(\mathbb{R})$ for $i=1,2$. Secondly, the solutions $w_{i}$ are in $C_{b}\left(0, \infty ; L^{2}(\mathbb{R})\right)$ and are continuous everywhere in the upper-half plane except that the points $\left(0, t_{i}\right)$, $i=1,2$, where $t_{1}=1 / \beta_{1} \alpha_{+}$and $t_{2}=1 / \beta_{2} \alpha_{-}$. Thus, it behooves us to choose $\beta_{1} / t^{*} \alpha_{+}$ and $\beta_{2}=1 / t^{*} \alpha_{-}$so that both $w_{1}$ and $w_{2}$ loose continuity and blow up at the same point $\left(0, t^{*}\right)$ in space-time. It follows that if $\left(u_{0}, v_{0}\right)$ is constructed from $\left(w_{1}(\cdot, 0)\right.$, $\left.w_{2}(\cdot, 0)\right)$ and $(\tilde{u}, \tilde{v})$ from $\left(w_{1}, w_{2}\right)$ via the transformation in $(4.7)$, then

$$
u_{0}, v_{0} \in L^{2}\left(\mathbb{R} ; w_{\sigma}\right) \cap C_{b}(\mathbb{R}) \cap C^{\infty}(\mathbb{R})
$$

for any $\sigma<m-\frac{1}{8}$, and $\tilde{u}$ and $\tilde{v}$ both have the blow-up property in (6.8) for $k=0$ at the point $\left(0, t^{*}\right)$. As $m>\frac{3}{16}$, it follows that $u_{0}, v_{0} \in L^{2}\left(\mathbb{R} ; w_{\sigma}\right)$ for values of $\sigma>\frac{1}{16}$, and so according to Proposition 6.4, the solution pair $(u, v)$ of (5.1) corresponding to the initial data $\left(u_{0}, v_{0}\right)$ lies in $L^{\infty}\left(0, T ; L^{2}\left(\mathbb{R}, w_{\sigma}\right)\right) \times L^{\infty}\left(0, T ; L^{2}\left(\mathbb{R}, w_{\sigma}\right)\right)$ for such values of $\sigma$. Appeal is again made to Duhamel's principle to write $(u, v)$ in the form expressed in (5.8), namely

$$
(u, v)(t)=(\tilde{u}, \tilde{v})(t)+\int_{0}^{t} W(t-\tau)\left(f_{1}, f_{2}\right)(\tau) d \tau
$$


where $(\tilde{u}, \tilde{v})$ is our current notation for $W(t)\left(u_{0}, v_{0}\right)$ and recall that $W$ is the linear semi-group generated by ignoring the nonlinear terms in (5.1).

Attention now focuses upon the second term on the right-hand side of (6.16). Each component of this integral is a sum of two terms that have the general form

$$
\int_{0}^{t} \int_{-\infty}^{\infty} \frac{1}{[a(t-s)]^{1 / 3}} A i\left(\frac{x-y}{[a(t-s)]^{1 / 3}}\right) \partial_{y} P(u, v) d y d s
$$

where $A i$ is the Airy function again, $a$ is a positive constant and $P$ is a polynomial in $u$ and $v$ each of whose terms is exactly quadratic. After an integration by parts in the variable $y$, we are presented with integrals of the form

$$
\int_{0}^{t} \frac{1}{[a(t-s)]^{2 / 3}} \int_{-\infty}^{\infty} A i^{\prime}\left(\frac{x-y}{[a(t-s)]^{1 / 3}}\right) P(u, v) d y d s .
$$

Because the functions $u$ and $v$ both lie in $L^{\infty}\left(0, T ; L^{2}\left(\mathbb{R} ; w_{\sigma}\right)\right)$ for $\sigma=\frac{1}{16}$ at least, it follows that the inner integral above is majorized by

$$
C\left\|\frac{A i^{\prime}\left(\frac{x-y}{[a(t-s)]^{1 / 3}}\right)}{\left(1+y^{2}\right)^{1 / 6}}\right\|_{L^{\infty}(d y)}\left(\|u\|_{L^{\infty}\left(0, T ; L^{2}(\mathbb{R} ; w)\right)}^{2}+\|v\|_{L^{\infty}\left(0, T ; L^{2}(\mathbb{R} ; w)\right)}^{2}\right) .
$$

This quantity is easily determined to be a locally bounded function of $(x, t)$ in the domain $\mathbb{R} \times \mathbb{R}^{+}$. Hence after performing the temporal integration, we are left with a continuous function of $(x, t)$ just as in the proof of Theorem 3.1 in Bona and Saut (1991à).

This latter deduction combined with the already established properties of $(\tilde{u}, \tilde{v})$ completes the proof of the theorem in the case $k=0$. The proof for $k>0$ follows very similar lines and so is omitted.

Remark 6.5. The results contained in this paper are easily seen to hold in a somewhat more general context, as already hinted in the last proof. In particular, the global existence of smooth solutions, global existence in $H^{1}$, existence in $L^{2}$, and dispersive blow up are all valid for a class of gradient system of the following form:

$$
\begin{aligned}
& u_{t}+a u_{x x x}+b v_{x x x}+\frac{\partial}{\partial x}\left[\frac{\partial}{\partial u} I(u, v)\right]=0 \\
& v_{t}+c u_{x x x}+d v_{x x x}+\frac{\partial}{\partial_{x}}\left[\frac{\partial}{\partial v} I(u, v)\right]=0
\end{aligned}
$$

where the functional $I$ is given by

$$
I(u, v)=\int_{-\infty}^{\infty} P(u, v) d x
$$


and $P$ is a polynomial in $u, v$ composed of terms of degree at most five, and where it is assumed that $(a+d)^{2}+4(b c-a d)>0$.

\section{References}

1. Alkylas, T. R., Benney, D. J.: Direct resonance in nonlinear wave systems. Stud. Appl. Math. 63, 209-226 (1980)

2. Alkylas, T. R., Benney, D. J.: The evolution of waves near direct-resonance conditions. Stud. Appl. Math. 67, 107-123 (1982)

3. Benjamin, T. B.: Internal waves of finite amplitude and permanent form. J. Fluid Mech. 25, 241-270 (1966)

4. Benjamin, T. B.: Internal waves of permanent form in fluids of great depth. J. Fluid Mech. 29, 559-592 (1967)

5. Bona, J. L., Sachs, R.: Global existence of smooth solutions and stability of solitary waves for a generalized Boussinesq equation. Commun. Math. Phys. 118, 15-29 (1988)

6. Bona, J. L., Saut, J.-C.: Dispersive blow-up for the generalized Korteweg-de Vries equation. To appear in J. Diff'l Equations (1991a)

7. Bona, J. L., Saut, J.-C.: The general intermediate long wave equation and related systems. In preparation (1991b)

8. Bona, J. L., Smith, R.: The initial value problem for the Korteweg-de Vries equation. Phil. Trans. R. Soc. Lond. A278, 555-604 (1975)

9. Erkart, C.: Internal waves in the ocean. Phys. Fluid 4, 791-799 (1961)

10. Fornberg, R. B., Whitham, G. B.: A numerical and theoretical study of certain nonlinear wave phenomena. Phil. Trans. R. Soc. Lond. A289, 373-404 (1978)

11. Gear, J. A.: Strong interactions between solitary waves belonging to different wave modes. Stud. Appl. Math. 72, 95-124 (1985)

12. Gear, J. A., Grimshaw, R.: Weak and strong interactions between internal solitary waves. Stud. Appl. Math. 70, 235-258 (1984)

13. Ginibre, J., Tsutsumi, Y.: Uniqueness for the generalized Korteweg-de Vries equation. SIAM J. Math. Anal. 20, 1388-1425 (1989)

14. Grimshaw, R.: Evolution equations for long, nonlinear internal waves in stratified shear flows. Stud. Appl. Math. 65, 159-188 (1981)

15. Kato, T.: Quasilinear equations of evolution with applications to partial differential equations, Lect. Notes in Math. 448, pp. 27-50. Berlin, Heidelberg, New York: Springer 1975

16. Kato, T.: On the Korteweg-de Vries equation. Manuscripta Math. 28, 89-99 (1979)

17. Kato, T.: On the Cauchy problem for the (generalized) Korteweg-de Vries equation. Adv. Math. Supplementary Studies in Applied Math. Vol. 8, pp.93-128 (1983)

18. Kato, T., Ponce, G.: Commutator estimates and the Euler and Navier-Stokes equations. Commun. Pure Applied Math. 41, 891-907 (1988)

19. Kenig, C. E., Ponce, G., Vega, L.: On the (generalized) Korteweg-de Vries equation. Duke Math. J. 59, 585-610 (1989)

20. Kenig, C. E., Ponce, G., Vega, L.: Oscillatory integrals and regularity of dispersive equations. Indiana U. Math. J. 40, 33-69 (1991a)

21. Kenig, C. E., Ponce, G., Vega, L.: Well-posedness of the initial value problem for the Korteweg-de Vries equation. J. Am. Math. Soc. 4, 323-347 (1991b)

22. Kenig, C. E., Ruiz, A.: A strong type $(2,2)$ estimate for the maximal function associated to the Schrödinger equation. Trans. Am. Math. Soc. 280, 239-246 (1983)

23. Kubota, T., Ko, D. R. S., Dobbs, L. D.: Weakly-nonlinear, long internal waves in stratified fluids of finite depth. AIAA J. Hydronautics 12, 157-165 (1980)

24. Lions, J.-L.: Quelques methodes de résolution des problèmes aux limites non linéaires. Paris: Dunod 1969

25. Lions, J.-L., Magenes, E.: Problèmes aux limites non homogènes et applications. Vol. 1. Paris: Dunod 1968

26. Liu, A. K., Kubota, T., Ko, D. R. S.: Resonant transfer of energy between nonlinear waves in neighboring pycnoclines. Stud. Appl. Math. 63, 25-45 (1980) 
27. Liu, A. K., Pereira, N. R., Ko, D. R. S.: Weakly interacting internal solitary waves in neighboring pycnoclines. J. Fluid Mech. 122, 187-194 (1982)

28. Saut, J.-C.: Sur quelques généralisations de l'équation de Korteweg-de Vries. J. Math. Pures Appl. 58, 21-61 (1975)

29. Saut, J.-C., Temam, R.: Remarks on the Korteweg-de Vries equation. Israel J. Math. 24, 78-87 (1976)

30. Stein, E. M.: Oscillatory integrals in Fourier Analysis. Beijing lectures in Harmonic Analysis, pp. 307-355. Princeton NJ: Princeton University Press 1986

31. Strichartz, R. S.: Restriction of Fourier transform to quadratic surfaces and decay of solutions of wave equations. Duke Math. J. 44, 705-714 (1977)

32. Vega, L.: El multiplicador de Schrödinger: La funcion maximal y los operadores de restriction. Doctoral Thesis Universidad Autonoma, Madrid, Spain 1987

Communicated by S.-T. Yau 
